\title{
Baryon electric dipole moments from strong CP violation
}

\author{
Feng-Kun Guo ${ }^{a}$ and Ulf-G. Meißner ${ }^{a, b}$ \\ ${ }^{a}$ Helmholtz-Institut für Strahlen- und Kernphysik and Bethe Center for Theoretical Physics, Universität \\ Bonn, D-53115 Bonn, Germany \\ ${ }^{b}$ Institute for Advanced Simulation, Institut für Kernphysik and Jülich Center for Hadron Physics, JARA- \\ FAME and JARA-HPC, Forschungszentrum Jülich, D-52425 Jülich, Germany \\ E-mail: fkguo@hiskp.uni-bonn.de, meissner@hiskp.uni-bonn.de
}

\begin{abstract}
The electric dipole form factors and moments of the ground state baryons are calculated in chiral perturbation theory at next-to-leading order. We show that the baryon electric dipole form factors at this order depend only on two combinations of low-energy constants. We also derive various relations that are free of unknown low-energy constants. We use recent lattice QCD data to calculate all baryon EDMs. In particular, we find $d_{n}=-2.9 \pm 0.9$ and $d_{p}=1.1 \pm 1.1$ in units of $10^{-16} e \theta_{0} \mathrm{~cm}$. Finite volume corrections to the electric dipole moments are also worked out. We show that for a precision extraction from lattice QCD data, the next-to-leading order terms have to be accounted for.
\end{abstract}

KEYWORDS: Chiral Lagrangians, CP violation, Lattice QCD 


\section{Contents}

1 Introduction 1

2 Effective Lagrangian for strong CP violation 3

3 Baryon electric dipole form factors in the infinite volume 5

$\begin{array}{lll}3.1 & \text { Baryon electric dipole form factors up to NLO } & 6\end{array}$

3.2 Numerical results for the loop contributions of the baryon EDMs 8

$\begin{array}{lr}3.3 \text { A first determination of the LECs from lattice results } & 12\end{array}$

$\begin{array}{lll}3.4 & \text { Counterterm-free relations } & 13\end{array}$

4 Finite-volume corrections in the $p$-regime $\quad 15$

$\begin{array}{lll}5 & \text { Summary } & 18\end{array}$

$\begin{array}{ll}\text { A Baryon masses up to NLO } & 20\end{array}$

B Loop integrals in infrared regularization $\quad 20$

C Expressions for the baryon EDFFs up to NLO 21

$\begin{array}{ll}\text { D Finite volume corrections to loops } & 24\end{array}$

E Asymptotic expansion of finite volume corrections 25

\section{Introduction}

The neutron electric dipole moment $(\mathrm{EDM})$ is a sensitive probe of $\mathrm{CP}$ violation in the Standard Model (SM) and beyond. The current experimental limit $\left|d_{n}\right| \leq 2.9 \cdot 10^{-26} e \mathrm{~cm}[1]$ is still orders of magnitude larger than the SM prediction due to weak interactions. Furthermore, in quantum chromodynamics (QCD) the breaking of the $\mathrm{U}(1)_{A}$ anomaly allows for strong $\mathrm{CP}$ violation, which is parameterized through the vacuum angle $\theta_{0}$. Therefore, an upper bound on $d_{n}$ allows to constrain the magnitude of $\theta_{0}$. Furthermore, such electric dipole moments are very sensitive to physics beyond the SM, see e.g. [2]. Many extensions of the SM in fact lead to larger EDMs than the tiny SM predictions, so that any limit of $d_{n}$ leads to bounds on the scale of the new physics. In this paper, we concentrate on the $\mathrm{CP}$ violation generated by the $\theta$-term of QCD. New and on-going experiments with ultracold neutrons strive to improve the aforementioned bounds even further, see e.g. [3] for a recent review. Furthermore, there are new experimental proposals to measure the EDM of the proton and the deuteron in storage rings, which in principle allow for an even higher sensitivity than obtained with the instable neutron, see e.g. refs. [4-7]. 
Besides these challenging experimental activities, first full lattice QCD calculations of the neutron and the proton electric dipole moment are becoming available. There exist three different methods of calculating the nucleon EDM on the lattice. The EDM can be related to the energy difference of the nucleon with different spin alignments in the presence of an external electric field [8-11]. It can also be obtained by calculating the electric dipole form factor (EDFF) at finite momentum transfer $q^{2}$, see the definition in eq. (3.1), and extrapolating to the point with $q^{2}=$ $0[12,13]$,

$$
d_{N}=\frac{F_{3, N}(0)}{2 m_{N}} \text {. }
$$

In addition, the nucleon EDM can also be calculated by analytically continuing $\theta_{0}$ to a purely imaginary quantity $[14,15]$, as the QCD action in the presence of the $\theta$-term becomes real in Euclidean space. For a brief review of these methods, see ref. [16]. We do not discuss here another method [17] that relates certain Fock state components of the EDM to the Fock state expansion of the magnetic moment (derived in light-front QCD) [18] since it is not clear what these relations imply for the observable quantities [19, 20].

These lattice studies require a careful study of the quark mass dependence of the nucleon EDM to connect to the physical light quark masses. In addition, CP-violating atomic effects can be sensitive to the nuclear Schiff moment, which receives a contribution from the radius of the nucleon electric dipole form factor (EDFF), see e.g. [21]. It is thus of paramount interest to improve the existing calculations of these fundamental quantities in the framework of chiral perturbation theory (CHPT). In [22], the electric dipole moments of the neutron and the $\Lambda$ were calculated within the framework of $\mathrm{U}(3)_{L} \times \mathrm{U}(3)_{R}$ heavy-baryon chiral perturbation theory and an estimate for $\theta_{0}$ was given (for earlier works utilizing chiral Lagrangians, see [23-25]). In [26], the electric dipole form factor of the nucleon was analyzed to leading one-loop accuracy in chiral SU(2). In that calculation, the form factor originates entirely from the pion cloud. The strength of the form factor was shown to be proportional to a non-derivative and CP-violating pion-nucleon coupling $\bar{g}_{\pi N N}$ that was estimated from dimensional analysis in [26]. In ref. [27], the results of refs. [22, 26] were extended to higher order based on a covariant version of $\mathrm{U}(3)_{L} \times \mathrm{U}(3)_{R}$ baryon CHPT, and an expression for $\bar{g}_{\pi N N}$ was given in terms of the measurable quantities. For other recent work on these issues, see [20, 28]. Here, we extend the studies of ref. [27] to the baryon octet and make contact to recent lattice QCD studies.

Furthermore, the leading contributions to the neutron EDM at finite volume and in partiallyquenched calculations were considered in [29], and in [30] the leading order extrapolation formula using a mixed action chiral Lagrangian is given. Here, we work out the finite volume expression for the whole baryon octet at next-to-leading order (NLO). In particular, we show that the formally suppressed NLO corrections are sizeable, and even dominate for some baryons due to large cancellations at leading order.

The manuscript is organized as follows. The underlying effective chiral $\mathrm{U}(3)_{L} \times \mathrm{U}(3)_{R}$ Lagrangian is given in section 2. In section 3 we work out the baryon EDFFs and EDMs in the infinite volume for varying quark masses. We derive new relations for all baryon EDMs and show that these only depend on two combinations of unknown low-energy constants (LECs). Using recent lattice data, we can give predictions for all baryon EDMs. Then, in section 4, we calculate the finite volume corrections for the baryons at NLO and show that these NLO corrections are substan- 
tial and must be included in any extraction from lattice data. We end with a summary and outlook in section 5. Various technical aspects of our calculations are displayed in the appendices.

\section{Effective Lagrangian for strong $\mathrm{CP}$ violation}

The most general gauge-invariant and renormalizable Lagrangian for QCD is

$$
\mathcal{L}_{Q C D}=-\frac{1}{4} G_{\mu \nu}^{a} G^{a, \mu \nu}+\bar{q}(i \not D-\mathcal{M}) q+\theta \frac{g^{2}}{32 \pi^{2}} G_{\mu \nu}^{a} \tilde{G}^{a, \mu \nu}(a=1, \ldots, 8),
$$

with $G_{\mu \nu}^{a}$ the gluon field strength tensor and $\tilde{G}_{\mu \nu}^{a}=\varepsilon_{\mu \nu \lambda \sigma} G^{a, \lambda \sigma} / 2$ its dual, $q$ collects the quark fields of various flavors, $D_{\mu}$ is the gauge-covariant derivative, and $\mathcal{M}$ is the quark mass matrix. The last term is the so-called $\theta$-term, which breaks the $\mathrm{P}$ and $\mathrm{CP}$ symmetries. It is a consequence of the $\mathrm{U}(1)_{A}$ anomaly. Because the theta-term is related to chiral $\mathrm{U}(1)$ transformations of the quark fields (see, for instance, ref. [31]), only the combination

$$
\theta_{0}=\theta+\arg \operatorname{det} \mathcal{M}
$$

is a measurable quantity. In CHPT, one may treat the $\theta$-term using an external field $\theta(x)$, and the QCD Green functions can be obtained by expanding the generating functional around $\theta(x)=\theta_{0}$ with real quark masses. Under an axial U(1) transformation, one has

$$
\theta(x) \rightarrow \theta(x)-2 N_{f} \alpha,
$$

where $N_{f}$ is the number of flavors, and $\alpha=\left(\alpha_{R}-\alpha_{L}\right) / 2$. In the limit of infinitely large number of colors $N_{c}$, the $\mathrm{U}(1)_{A}$ anomaly is absent. In this case, the spontaneous chiral symmetry breaking of $\mathrm{U}(3)_{L} \times \mathrm{U}(3)_{R}$, which is a symmetry of the QCD Lagrangian, into $\mathrm{U}(3)_{V}$ gives nine Goldstone bosons (the $\mathrm{SU}(3)$ flavor octet $\left\{\pi^{ \pm}, \pi^{0}, K^{ \pm}, K^{0}, \bar{K}^{0}, \eta_{8}\right\}$ and the flavor singlet $\eta_{0}$ ). Collecting these fields in $\tilde{U}(x)$, this transforms under the axial $\mathrm{U}(1)$ transformation as

$$
\tilde{U}(x) \rightarrow e^{i \alpha_{R}} \tilde{U}(x) e^{-i \alpha_{L}} .
$$

Thus, the combination

$$
\bar{\theta}(x)=\theta(x)-i \ln \operatorname{det} \tilde{U}(x),
$$

is invariant under chiral transformations.

With $\bar{\theta}(x)$, the most general chiral effective Lagrangian which is invariant under $\mathrm{U}(3)_{L} \times \mathrm{U}(3)_{R}$ can be constructed. The original construction at order $\mathcal{O}\left(\delta^{2}\right)$ can be found in [32], where $\mathcal{O}(\delta)=$ $\mathcal{O}\left(M_{\phi}, k\right)$ with $M_{\phi}$ and $k$ denoting the Goldstone boson masses and a small momentum, respectively. Further, we count $1 / N_{c}$ as $\mathcal{O}\left(\delta^{2}\right)$. Here, we adopt the notation used in [22], whose formulation is partially based on refs. [33, 34]. The most general chiral effective Lagrangian for mesons to second chiral order, complying with the $\mathrm{U}(3)_{L} \times \mathrm{U}(3)_{R}$ symmetry, reads

$$
\begin{aligned}
\mathcal{L}= & -V_{0}+V_{1} \operatorname{Tr}\left[\nabla_{\mu} \tilde{U}^{\dagger} \nabla^{\mu} \tilde{U}\right]+V_{2} \operatorname{Tr}\left[\tilde{\chi}^{\dagger} \tilde{U}+\tilde{\chi} \tilde{U}^{\dagger}\right]+i V_{3} \operatorname{Tr}\left[\tilde{\chi}^{\dagger} \tilde{U}-\tilde{\chi} \tilde{U}^{\dagger}\right] \\
& +V_{4} \operatorname{Tr}\left[\tilde{U} \nabla_{\mu} \tilde{U}^{\dagger}\right] \operatorname{Tr}\left[\tilde{U}^{\dagger} \nabla^{\mu} \tilde{U}\right]+V_{5} \operatorname{Tr}\left[\nabla_{\mu} \theta \nabla^{\mu} \theta\right]
\end{aligned}
$$

where $\tilde{\chi}=2 B_{0} M_{q}$ with $M_{q}=\operatorname{diag}\left(m_{u}, m_{d}, m_{s}\right)$ the real quark mass matrix, $\nabla_{\mu} \tilde{U}=\partial_{\mu} \tilde{U}-$ $i r_{\mu} \tilde{U}+i \tilde{U} l_{\mu}$, and the $V_{i}$ 's are functions of $\bar{\theta}(x)$. 
In order to use the above Lagrangian, we need to determine the vacuum. Denoting the vacuum expectation value of $\tilde{U}$ by $U_{0}$, we can decompose $\tilde{U}$ as

$$
\tilde{U}=\sqrt{U_{0}} U \sqrt{U_{0}}
$$

where

$$
U=\exp \left(i \sqrt{\frac{2}{3}} \frac{\eta_{0}}{F_{0}}+i \frac{\sqrt{2}}{F_{\pi}} \phi\right)
$$

with

$$
\phi=\left(\begin{array}{ccc}
\frac{1}{\sqrt{2}} \pi^{0}+\frac{1}{\sqrt{6}} \eta_{8} & \pi^{+} & K^{+} \\
\pi^{-} & -\frac{1}{\sqrt{2}} \pi^{0}+\frac{1}{\sqrt{6}} \eta_{8} & K^{0} \\
K^{-} & \bar{K}^{0} & -\frac{2}{\sqrt{6}} \eta_{8}
\end{array}\right) .
$$

When $\theta_{0}=0$, the vacuum is trivial, $U_{0}=1$. When the $\theta_{0}$ angle is switched on, the vacuum is shifted, and $U_{0}$ has to be determined by minimizing the zero modes of the Lagrangian. One may parametrize the vacuum as

$$
U_{0}=\operatorname{diag}\left(e^{-i \varphi_{u}}, e^{-i \varphi_{d}}, e^{-i \varphi_{s}}\right)
$$

After the vacuum alignment, one obtains the effective Lagrangian [22] ${ }^{1}$

$$
\begin{aligned}
\mathcal{L}_{\phi}= & -V_{0}+V_{1} \operatorname{Tr}\left[\nabla_{\mu} U^{\dagger} \nabla^{\mu} U\right]+\left(V_{2}+\mathcal{B} V_{3}\right) \operatorname{Tr}\left[\chi\left(U+U^{\dagger}\right)\right]-i \mathcal{A} V_{2} \operatorname{Tr}\left[U-U^{\dagger}\right] \\
& +\mathcal{A} V_{3} \operatorname{Tr}\left[U+U^{\dagger}\right]+V_{4} \operatorname{Tr}\left[U \nabla_{\mu} U^{\dagger}\right] \operatorname{Tr}\left[U^{\dagger} \nabla^{\mu} U\right]
\end{aligned}
$$

where $\chi=2 B_{0} \operatorname{diag}\left(m_{u} \cos \varphi_{u}, m_{d} \cos \varphi_{d}, m_{s} \cos \varphi_{s}\right)$, and $\mathcal{A}, \mathcal{B}$ are complicated functions of the $V_{i}$ 's, see e.g. ref. [22]. Introducing the notation $\bar{\theta}_{0}=\theta_{0}-\sum_{q} \varphi_{q}$, one may expand the $V_{i}$ 's in terms of $\bar{\theta}_{0}$. All of them except for $V_{3}$, which is an odd function, are even functions of $\bar{\theta}_{0}$. In the leading approximation, $\mathcal{A}$ and $\mathcal{B}$ are given by

$$
\mathcal{A}=\frac{V_{0}^{(2)}}{V_{2}^{(0)}} \bar{\theta}_{0}+\mathcal{O}\left(\delta^{4}\right), \quad \mathcal{B}=\frac{V_{3}^{(1)}}{V_{2}^{(0)}} \bar{\theta}_{0}+\mathcal{O}\left(\delta^{6}\right) .
$$

Since

$$
\ln \operatorname{det} U=\operatorname{Tr} \ln U=i \frac{\sqrt{6}}{F_{0}} \eta_{0},
$$

the $V_{i}$ are now functions of $\bar{\theta}_{0}+\sqrt{6} \eta_{0} / F_{0}$. The correct normalization of the kinetic terms of the Goldstone boson fields can be obtained by requiring

$$
V_{1}(0)=V_{2}(0)=\frac{F_{\pi}^{2}}{4}, \quad V_{4}(0)=\frac{1}{12}\left(F_{0}^{2}-F_{\pi}^{2}\right) .
$$

Finally, the quantity $\bar{\theta}$ can be expressed in terms of the measurable quantity $\theta_{0}$ [27],

$$
\bar{\theta}_{0}=\left[1+\frac{4 V_{0}^{(2)}}{F_{\pi}^{2}} \frac{4 M_{K}^{2}-M_{\pi}^{2}}{M_{\pi}^{2}\left(2 M_{K}^{2}-M_{\pi}^{2}\right)}\right]^{-1} \theta_{0} .
$$

\footnotetext{
${ }^{1}$ In ref. [22], there is one more term $i\left(V_{3}-\mathcal{B} V_{2}\right) \operatorname{Tr}\left[\chi\left(U-U^{\dagger}\right)\right]$. However, this term vanishes exactly because $\mathcal{B}$ is defined as $V_{3} / V_{2}$.
} 
One sees that $\bar{\theta}_{0}=\mathcal{O}\left(\delta^{2}\right)$, because $V_{0}^{(2)}$ is of the zeroth chiral order.

Similarly, one can also construct the most general effective Lagrangian in $\mathrm{U}(3)_{L} \times \mathrm{U}(3)_{R}$ CHPT for the baryon octet (for a general discussion of effective Lagrangians for the $\theta$-term, see [20])

$$
B=\left(\begin{array}{ccc}
\frac{1}{\sqrt{2}} \Sigma^{0}+\frac{1}{\sqrt{6}} \Lambda & \Sigma^{+} & p \\
\Sigma^{-} & -\frac{1}{\sqrt{2}} \Sigma^{0}+\frac{1}{\sqrt{6}} \Lambda & n \\
\Xi^{-} & \Xi^{0} & -\frac{2}{\sqrt{6}} \Lambda
\end{array}\right) .
$$

The Lagrangian up to the second chiral order is (only the terms relevant to our calculation are displayed; for details, see ref. [22])

$$
\begin{aligned}
\mathcal{L}_{\phi B}= & i \operatorname{Tr}\left[\bar{B} \gamma^{\mu}\left[D_{\mu}, B\right]\right]-\stackrel{\circ}{m} \operatorname{Tr}[\bar{B} B]-\frac{D}{2} \operatorname{Tr}\left[\bar{B} \gamma^{\mu} \gamma_{5}\left\{u_{\mu}, B\right\}\right]-\frac{F}{2} \operatorname{Tr}\left[\bar{B} \gamma^{\mu} \gamma_{5}\left[u_{\mu}, B\right]\right] \\
& +\frac{w_{0}}{2} \operatorname{Tr}\left[\bar{B} \gamma^{\mu} \gamma_{5} B\right] \operatorname{Tr}\left[u_{\mu}\right]+b_{D} \operatorname{Tr}[\bar{B}\{\tilde{\chi}+, B\}]+b_{F} \operatorname{Tr}\left[\bar{B}\left[\tilde{\chi}_{+}, B\right]\right]+b_{0} \operatorname{Tr}[\bar{B} B] \operatorname{Tr}\left[\tilde{\chi}_{+}\right] \\
& +4 \mathcal{A} w_{10}^{\prime} \frac{\sqrt{6}}{F_{0}} \eta_{0} \operatorname{Tr}[\bar{B} B]+i\left(w_{13}^{\prime} \bar{\theta}_{0}+w_{13} \frac{\sqrt{6}}{F_{0}} \eta_{0}\right) \operatorname{Tr}\left[\bar{B} \sigma^{\mu \nu} \gamma_{5}\left\{F_{\mu \nu}^{+}, B\right\}\right] \\
& +i\left(w_{14}^{\prime} \bar{\theta}_{0}+w_{14} \frac{\sqrt{6}}{F_{0}} \eta_{0}\right) \operatorname{Tr}\left[\bar{B} \sigma^{\mu \nu} \gamma_{5}\left[F_{\mu \nu}^{+}, B\right]\right]
\end{aligned}
$$

where $\tilde{\chi}_{+}=\chi_{+}-i \mathcal{A}\left(U-U^{\dagger}\right)$, and we use the same notation $w_{10}^{\prime}=w_{10}+3 w_{12} / 2$ as in ref. [27] with the low-energy constants (LECs) $w_{10}$ and $w_{12}$ defined in ref. [22]. We remark here that although there seems to be quite a number of unknown LECs, i.e. $w_{0}, w_{10}^{\prime}, w_{13}, w_{13}^{\prime}, w_{14}$ and $w_{14}^{\prime}$, only two combinations of these will finally appear in the expressions of the baryon EDFFs.

\section{Baryon electric dipole form factors in the infinite volume}

The electromagnetic form factors of a baryon are defined by

$$
\begin{aligned}
\left\langle B\left(p^{\prime}\right)\left|J_{\mathrm{em}}^{\nu}\right| B(p)\right\rangle= & \bar{u}\left(p^{\prime}\right)\left[\gamma^{\nu} F_{1}\left(q^{2}\right)-\frac{i F_{2}\left(q^{2}\right)}{2 m_{B}} \sigma^{\mu \nu} q_{\mu}\right. \\
& \left.+i\left(\gamma^{\nu} q^{2} \gamma_{5}-2 m_{B} q^{\nu} \gamma_{5}\right) F_{A}\left(q^{2}\right)-\frac{F_{3}\left(q^{2}\right)}{2 m_{B}} \sigma^{\mu \nu} q_{\mu} \gamma_{5}\right] u(p),
\end{aligned}
$$

where $F_{1}\left(q^{2}\right)$ and $F_{2}\left(q^{2}\right)$ are the P-and CP-conserving Dirac and Pauli form factors, respectively, while $F_{A}\left(q^{2}\right)$ is the P-violating anapole form factor, and $F_{3}\left(q^{2}\right)$ if the electric dipole form factor (EDFF) which breaks $\mathrm{P}$ and $\mathrm{CP}$ symmetries. Here, $J_{\mathrm{em}}^{\nu}$ is the electromagnetic current, the baryon mass is denoted by $m_{B}$, and $q_{\mu}=p_{\mu}^{\prime}-p_{\mu}$ is the four-momentum transfer.

In what follows, we will consider the dipole form factor $F_{3}\left(q^{2}\right)$. The electric dipole moment of a baryon is defined as the electric dipole form factor at $q^{2}=0$

$$
d_{B}=\frac{F_{3, B}(0)}{2 m_{B}} .
$$

Note that similar to the case of the neutron electric form factor, we do not include the normalization of the form factor at $q^{2}=0$ in this definition. 


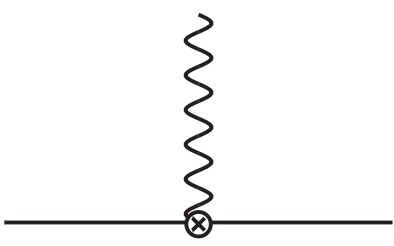

(a)

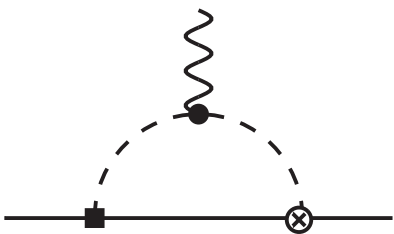

(d)

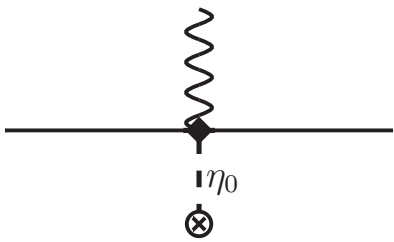

(b)

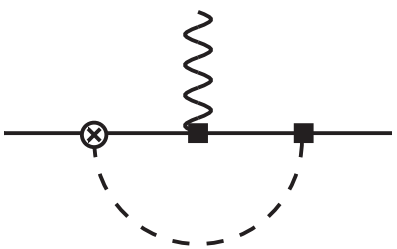

(e)

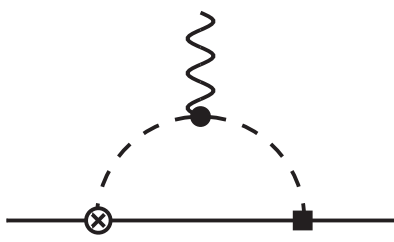

(c)

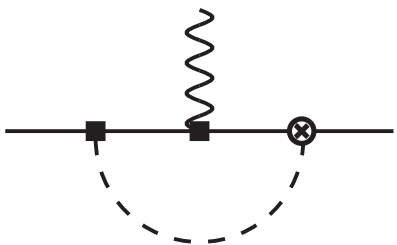

(f)

Figure 1. Feynman diagrams contributing to the baryon EDFFs up to next-to-leading order, where $\otimes$ denotes a CP violating vertex, black dots represent the second order mesonic vertices, filled squares and diamonds are the first and second order baryonic vertices, respectively.

\begin{tabular}{|l|c|}
\hline Baryons & Tree-level \\
\hline$p$ & $-\frac{4}{3} e \bar{\theta}_{0}\left[\alpha\left(w_{13}+3 w_{14}\right)+w_{13}^{\prime}+3 w_{14}^{\prime}\right]$ \\
$n$ & $\frac{8}{3} e \bar{\theta}_{0}\left(\alpha w_{13}+w_{13}^{\prime}\right)$ \\
$\Lambda$ & $\frac{4}{3} e \bar{\theta}_{0}\left(\alpha w_{13}+w_{13}^{\prime}\right)$ \\
$\Sigma^{+}$ & $-\frac{4}{3} e \bar{\theta}_{0}\left[\alpha\left(w_{13}+3 w_{14}\right)+w_{13}^{\prime}+3 w_{14}^{\prime}\right]$ \\
$\Sigma^{0}$ & $-\frac{4}{3} e \bar{\theta}_{0}\left(\alpha w_{13}+w_{13}^{\prime}\right)$ \\
$\Sigma^{-}$ & $-\frac{4}{3} e \bar{\theta}_{0}\left[\alpha\left(w_{13}-3 w_{14}\right)+w_{13}^{\prime}-3 w_{14}^{\prime}\right]$ \\
$\Xi^{0}$ & $\frac{8}{3} e \bar{\theta}_{0}\left(\alpha w_{13}+w_{13}^{\prime}\right)$ \\
$\Xi^{-}$ & $-\frac{4}{3} e \bar{\theta}_{0}\left[\alpha\left(w_{13}-3 w_{14}\right)+w_{13}^{\prime}-3 w_{14}^{\prime}\right]$ \\
\hline
\end{tabular}

Table 1. Tree-level contribution to the EDFFs of the octet baryons.

\subsection{Baryon electric dipole form factors up to NLO}

Consider first the tree level contributions to the baryon EDFFs, figure 1(a,b). In particular, these feature the counterterms $w_{13,14}^{\prime}$ and $w_{13,14}$ at $\mathcal{O}\left(\delta^{2}\right)[22,27]$. The expressions of the form factor $F_{3}\left(q^{2}\right) /(2 m)$ of the ground state octet baryons from the tree-level diagrams are collected in table $1,{ }^{2}$ where $\alpha=144 V_{0}^{(2)} V_{3}^{(1)} /\left(F_{0} F_{\pi} M_{\eta_{0}}\right)^{2}$. One notices that the tree-level contributions to various baryons depend only on two combinations of the LECs: $\alpha w_{13}+w_{13}^{\prime}$ and $\alpha w_{14}+w_{14}^{\prime}$. The dependence on the latter may be written in the general form $-4 Q_{B} e \bar{\theta}_{0}\left(\alpha w_{14}+w_{14}^{\prime}\right)$, with $Q_{B}$ the baryon electric charge.

There are four loop graphs contributing to the baryon EDFFs up to NLO, see figure 1(c-f). Formally, there are more diagrams. However, due to cancellations they only contribute starting at

\footnotetext{
${ }^{2}$ The overall sign of the tree-level expression for the neutron EDM given in ref. [27] should be positive.
} 


\begin{tabular}{|l|c|cc|}
\hline Baryons & Loops & $C_{\mathrm{cd}}$ & $C_{\mathrm{ef}}$ \\
\hline$n$ & $\left\{\pi^{-}, p\right\}$ & $2(D+F)\left(b_{D}+b_{F}\right)$ & $-2(D+F)\left(b_{D}+b_{F}\right)$ \\
& $\left\{K^{+}, \Sigma^{-}\right\}$ & $-2(D-F)\left(b_{D}-b_{F}\right)$ & $2(D-F)\left(b_{D}-b_{F}\right)$ \\
\hline$p$ & $\left\{\pi^{0}, p\right\}$ & 0 & $-(D+F)\left(b_{D}+b_{F}\right)$ \\
& $\left\{\pi^{+}, n\right\}$ & $-2(D+F)\left(b_{D}+b_{F}\right)$ & 0 \\
& $\left\{K^{0}, \Sigma^{+}\right\}$ & 0 & $-2(D-F)\left(b_{D}-b_{F}\right)$ \\
& $\left\{K^{+}, \Lambda\right\}$ & $-\frac{1}{3}(D+3 F)\left(b_{D}+3 b_{F}\right)$ & 0 \\
& $\left\{K^{+}, \Sigma^{0}\right\}$ & $-(D-F)\left(b_{D}-b_{F}\right)$ & 0 \\
& $\left\{\eta_{8}, p\right\}$ & 0 & $-\frac{1}{3}(D-3 F)\left(b_{D}-3 b_{F}\right)$ \\
& $\left\{\eta_{0}, p\right\}$ & 0 & $-\frac{2 F_{\pi}^{2}}{3 F_{0}^{2}} \beta$ \\
\hline
\end{tabular}

Table 2. Possible loops contributing to the EDFFs of the nucleons and the corresponding coefficients $C_{\mathrm{cd}}$ and $C_{\text {ef }}$. The intermediate states of the loops are listed in the second column for each nucleon.

next-to-next-to-leading order. For details, see refs. [27, 35]. The expression for the sum of loops of a given pair of any meson-baryon intermediate state is

$$
\begin{aligned}
\frac{F_{3}^{M \tilde{m}}\left(q^{2}\right)}{2 m}= & \frac{8 e \bar{\theta}_{0} V_{0}^{(2)}}{F_{\pi}^{4}}\left\{C_{\mathrm{cd}}\left[-J_{M M}\left(q^{2}\right)+\left(2 m(m-\tilde{m})+M^{2}-\frac{q^{2}}{2}\right) J_{M M \tilde{m}}\left(q^{2}, m^{2}\right)\right]\right. \\
& \left.+\left(C_{\mathrm{cd}}+C_{\mathrm{ef}}\right) J_{M \tilde{m}}\left(m^{2}\right)\right\}+\mathcal{O}\left(\delta^{4}\right)
\end{aligned}
$$

where $m$ is the mass of the external baryon, $M$ and $\tilde{m}$ are the masses of the meson and baryon in the loops, respectively. We have made use of the fact that the baryon mass difference is $\mathcal{O}\left(\delta^{2}\right)$, see appendix A. Keeping only the leading order, the loop expression does not depend on the baryon mass, and reads

$$
\frac{F_{3 \mathrm{LO}}^{M \tilde{m}}\left(q^{2}\right)}{2 m}=-\frac{8 e \bar{\theta}_{0} V_{0}^{(2)}}{F_{\pi}^{4}} C_{\mathrm{cd}} J_{M M}\left(q^{2}\right)
$$

The expressions for the loop functions involved in eqs. (3.3) and (3.4), making use of infrared regularization [36], are collected in appendix B.

Different baryons receive contributions from loops with different intermediate states. For instance, the loops for the neutron can be $\left\{\pi^{-}, p\right\}$ and $\left\{K^{+}, \Sigma^{-}\right\}$, while they are $\left\{\pi^{+}, n\right\},\left\{\pi^{0}\left(\eta_{8}, \eta_{0}\right), p\right\}$, $\left\{K^{+}, \Sigma^{0}(\Lambda)\right\}$ and $\left\{K^{0}, \Sigma^{+}\right\}$for the proton. A list of the possible intermediate states and the corresponding coefficients $C_{\mathrm{cd}}$ and $C_{\mathrm{ef}}$ for the nucleons are given in table 2, where we have defined

$$
\beta=\left(2 D-3 w_{0}\right)\left(2 b_{D}+3 b_{0}+6 w_{10}^{\prime}\right)
$$

for brevity. The loops and coefficients for the $\Sigma, \Lambda$ and $\Xi$ hyperons are collected in table 3 . The loops are divergent. Up to $\mathcal{O}\left(\delta^{3}\right)$, the divergences can be absorbed into the renormalization of $w_{13}^{\prime}$ 
and $w_{14}^{\prime}$,

$$
\begin{aligned}
& w_{13}^{\prime}=w_{13}^{\prime r}(\mu)+\frac{24 V_{0}^{(2)}}{F_{\pi}^{4}}\left(D b_{F}+F b_{D}\right) L, \\
& w_{14}^{\prime}=w_{14}^{\prime r}(\mu)+\frac{8 V_{0}^{(2)}}{3 F_{\pi}^{4}}\left(5 D b_{D}+9 F b_{F}\right) L,
\end{aligned}
$$

where the divergence is contained in

$$
L=\frac{\mu^{d-4}}{(4 \pi)^{2}}\left\{\frac{1}{d-4}-\frac{1}{2}\left[\ln (4 \pi)+\Gamma^{\prime}(1)+1\right]\right\},
$$

with $d$ the number of space-time dimension, and $w_{13,14}^{\prime r}(\mu)$ are the finite parts of $w_{13,14}^{\prime}$. Notice that $w_{13,14}^{\prime r}(\mu)$ depend on the renormalization scale $\mu$ through $L$. The scale dependence cancels with that of the loops, and as a result, the final expressions of the EDFFs are scale-independent. With the coefficients collected in tables 2 and 3, one can easily obtain the explicit expressions of the baryon EDFFs up to NLO. They are listed in appendix C.

So far, there is no constraint on the LECs $w_{13,14}^{\prime r}(\mu)$ and $w_{13,14}$, which appear at the LO of the baryon EDFFs, except for the $N_{c}$ scaling and the naturalness requirement of the effective field theory. As mentioned in section 3.1, the tree-level expression of the baryon EDFFs depend only on two combinations

$$
w_{a}(\mu) \equiv \alpha w_{13}+w_{13}^{\prime r}(\mu)
$$

and $\alpha w_{14}+w_{14}^{\prime r}(\mu)$. In principle, these two combinations may be extracted from lattice calculations of the baryon EDMs. On the lattice, the calculations can be performed at different quark masses, or equivalently pion masses. From a fitting to the pion mass dependence of some of the baryon EDMs, one may extract the unknown LECs, and then make a prediction of the other baryon EDMs. Furthermore, up to the order $\mathcal{O}\left(\delta^{3}\right)$,

$$
w_{b}(\mu) \equiv 3\left[\alpha w_{14}+w_{14}^{\prime r}(\mu)\right]+\frac{V_{0}^{(2)} \beta}{4 \pi F_{0}^{2} F_{\pi}^{2} m_{\text {ave }}} M_{\eta_{0}}
$$

always appear together in the expressions of the baryon EDFFs because of SU(3) flavor symmetry. The second term is due to the loops involving the $\eta_{0}$, and its pion mass dependence starts from $\mathcal{O}\left(\delta^{4}\right)$. Here, all the baryon masses in the second term have been replaced by the average mass of the baryons $m_{\text {ave }}$ since the difference is a higher order effect. Consequently, NLO one-loop expressions for all baryon EDFFs are given in terms of just two unknown LECs.

\subsection{Numerical results for the loop contributions of the baryon EDMs}

Since the values of the LECs $w_{13,14}^{\prime r}(\mu)$ and $w_{13,14}$ are not known, we will only focus on the contributions from the loops in this section, and discuss several relations which are free of these parameters in section 3.4. In order to get the numerical results, we use $D=0.804$ and $F=0.463$. From fitting to the baryon mass differences at $\mathcal{O}\left(\delta^{2}\right)$, see appendix A, we get $b_{D}=0.068 \mathrm{GeV}^{-1}$ and $b_{F}=-0.209 \mathrm{GeV}^{-1}$. There are other determinations of $b_{D}$ and $b_{F}$ from higher order analysis of various baryonic properties, see, for instance, refs. [37, 38]. The difference reflects higher order effects, so that they will not be used here. In the large $N_{c}$-limit, one has $F_{0}=F_{\pi}$, and 


\begin{tabular}{|c|c|c|c|}
\hline Baryons & Loops & $C_{\mathrm{cd}}$ & $C_{\mathrm{ef}}$ \\
\hline \multirow[t]{7}{*}{$\Sigma^{+}$} & $\left\{\pi^{0}, \Sigma^{+}\right\}$ & 0 & $-4 F b_{F}$ \\
\hline & $\left\{\pi^{+}, \Lambda\right\}$ & $-\frac{4}{3} D b_{D}$ & 0 \\
\hline & $\left\{\pi^{+}, \Sigma^{0}\right\}$ & $-4 F b_{F}$ & 0 \\
\hline & $\left\{\bar{K}^{0}, p\right\}$ & 0 & $-2(D-F)\left(b_{D}-b_{F}\right)$ \\
\hline & $\left\{K^{+}, \Xi^{0}\right\}$ & $-2(D+F)\left(b_{D}+b_{F}\right)$ & 0 \\
\hline & $\left\{\eta_{8}, \Sigma^{+}\right\}$ & 0 & $-\frac{4}{3} D b_{D}$ \\
\hline & $\left\{\eta_{0}, \Sigma^{+}\right\}$ & 0 & $-\frac{2 F_{\pi}^{2}}{3 F_{0}^{2}} \beta$ \\
\hline \multirow[t]{4}{*}{$\Sigma^{0}$} & $\left\{\pi^{+}, \Sigma^{-}\right\}$ & $-4 F B_{F}$ & $4 F B_{F}$ \\
\hline & $\left\{\pi^{-}, \Sigma^{+}\right\}$ & $4 F B_{F}$ & $-4 F B_{F}$ \\
\hline & $\left\{K^{+}, \Xi^{-}\right\}$ & $-(D+F)\left(b_{D}+b_{F}\right)$ & $(D+F)\left(b_{D}+b_{F}\right)$ \\
\hline & $\left\{K^{-}, p\right\}$ & $(D-F)\left(b_{D}-b_{F}\right)$ & $-(D-F)\left(b_{D}-b_{F}\right)$ \\
\hline \multirow[t]{7}{*}{$\Sigma^{-}$} & $\left\{\pi^{0}, \Sigma^{-}\right\}$ & 0 & $4 F b_{F}$ \\
\hline & $\left\{\pi^{-}, \Lambda\right\}$ & $\frac{4}{3} D b_{D}$ & 0 \\
\hline & $\left\{\pi^{-}, \Sigma^{0}\right\}$ & $4 F b_{F}$ & 0 \\
\hline & $\left\{K^{0}, \Xi^{-}\right\}$ & 0 & $2(D+F)\left(b_{D}+b_{F}\right)$ \\
\hline & $\left\{K^{-}, n\right\}$ & $2(D-F)\left(b_{D}-b_{F}\right)$ & 0 \\
\hline & $\left\{\eta_{8}, \Sigma^{-}\right\}$ & 0 & $\frac{4}{3} D b_{D}$ \\
\hline & $\left\{\eta_{0}, \Sigma^{-}\right\}$ & 0 & $\frac{2 F_{\pi}^{2}}{3 F_{0}^{2}} \beta$ \\
\hline \multirow[t]{4}{*}{$\Lambda$} & $\left\{\pi^{+}, \Sigma^{-}\right\}$ & $-\frac{4}{3} D b_{D}$ & $\frac{4}{3} D b_{D}$ \\
\hline & $\left\{\pi^{-}, \Sigma^{+}\right\}$ & $\frac{4}{3} D b_{D}$ & $-\frac{4}{3} D b_{D}$ \\
\hline & $\left\{K^{+}, \Xi^{-}\right\}$ & $-\frac{1}{3}(D-3 F)\left(b_{D}-3 b_{F}\right)$ & $\frac{1}{3}(D-3 F)\left(b_{D}-3 b_{F}\right)$ \\
\hline & $\left\{K^{-}, p\right\}$ & $\frac{1}{3}(D+3 F)\left(b_{D}+3 b_{F}\right)$ & $-\frac{1}{3}(D+3 F)\left(b_{D}+3 b_{F}\right)$ \\
\hline \multirow[t]{2}{*}{$\Xi^{0}$} & $\left\{\pi^{+}, \Xi^{-}\right\}$ & $-2(D-F)\left(b_{D}-b_{F}\right)$ & $2(D-F)\left(b_{D}-b_{F}\right)$ \\
\hline & $\left\{K^{-}, \Sigma^{+}\right\}$ & $2(D+F)\left(b_{D}+b_{F}\right)$ & $-2(D+F)\left(b_{D}+b_{F}\right)$ \\
\hline \multirow[t]{7}{*}{$\Xi^{-}$} & $\left\{\pi^{0}, \Xi^{-}\right\}$ & 0 & $(D-F)\left(b_{D}-b_{F}\right)$ \\
\hline & $\left\{\pi^{-}, \Xi^{0}\right\}$ & $2(D-F)\left(b_{D}-b_{F}\right)$ & 0 \\
\hline & $\left\{\bar{K}^{0}, \Sigma^{-}\right\}$ & 0 & $2(D+F)\left(b_{D}+b_{F}\right)$ \\
\hline & $\left\{K^{-}, \Lambda\right\}$ & $\frac{1}{3}(D-3 F)\left(b_{D}-3 b_{F}\right)$ & 0 \\
\hline & $\left\{K^{-}, \Sigma^{0}\right\}$ & $(D+F)\left(b_{D}+b_{F}\right)$ & 0 \\
\hline & $\left\{\eta_{8}, \Xi^{-}\right\}$ & 0 & $\left.\frac{1}{3} D+3 F\right)\left(b_{D}+3 b_{F}\right)$ \\
\hline & $\left\{\eta_{0}, \Xi^{-}\right\}$ & 0 & $\frac{2 F_{\pi}^{2}}{3 F_{0}^{2}} \beta$ \\
\hline
\end{tabular}

Table 3. Possible loops contributing to the EDFFs of the hyperons and the corresponding coefficients $C_{\mathrm{cd}}$ and $C_{\text {ef }}$. The intermediate states of the loops are listed in the second column for each hyperon. 

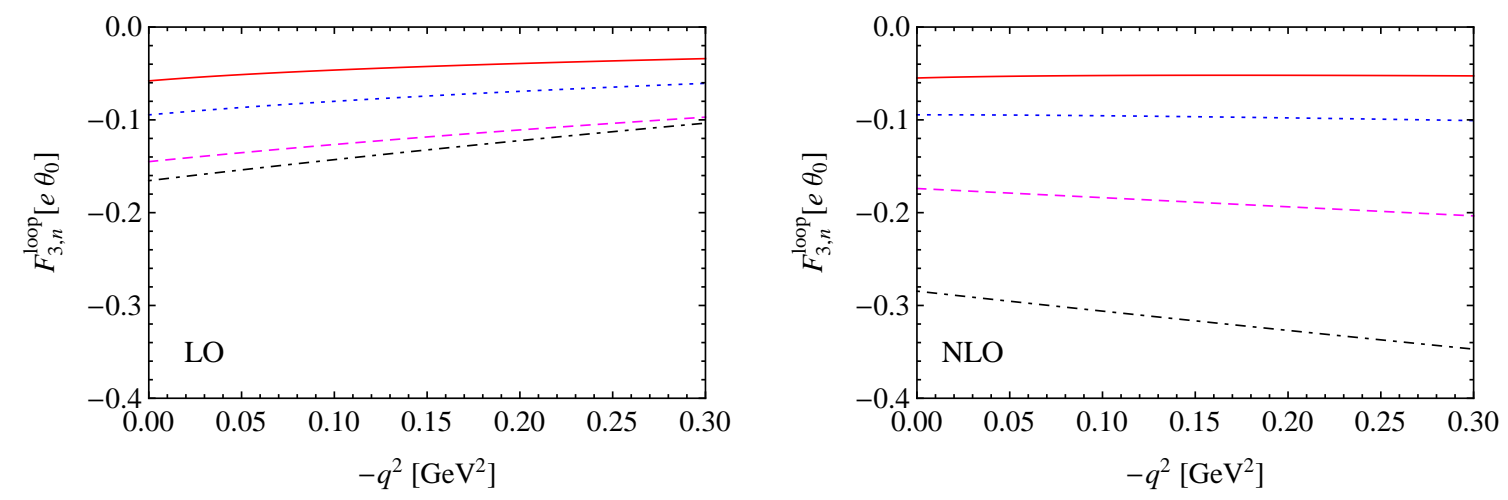

Figure 2. Loop contribution to the neutron EDFF up to LO (left) and NLO (right). The solid, dotted, dashed and dot-dashed lines are for the pion mass $138 \mathrm{MeV}$ (physical value), $200 \mathrm{MeV}, 300 \mathrm{MeV}$ and $400 \mathrm{MeV}$, respectively.
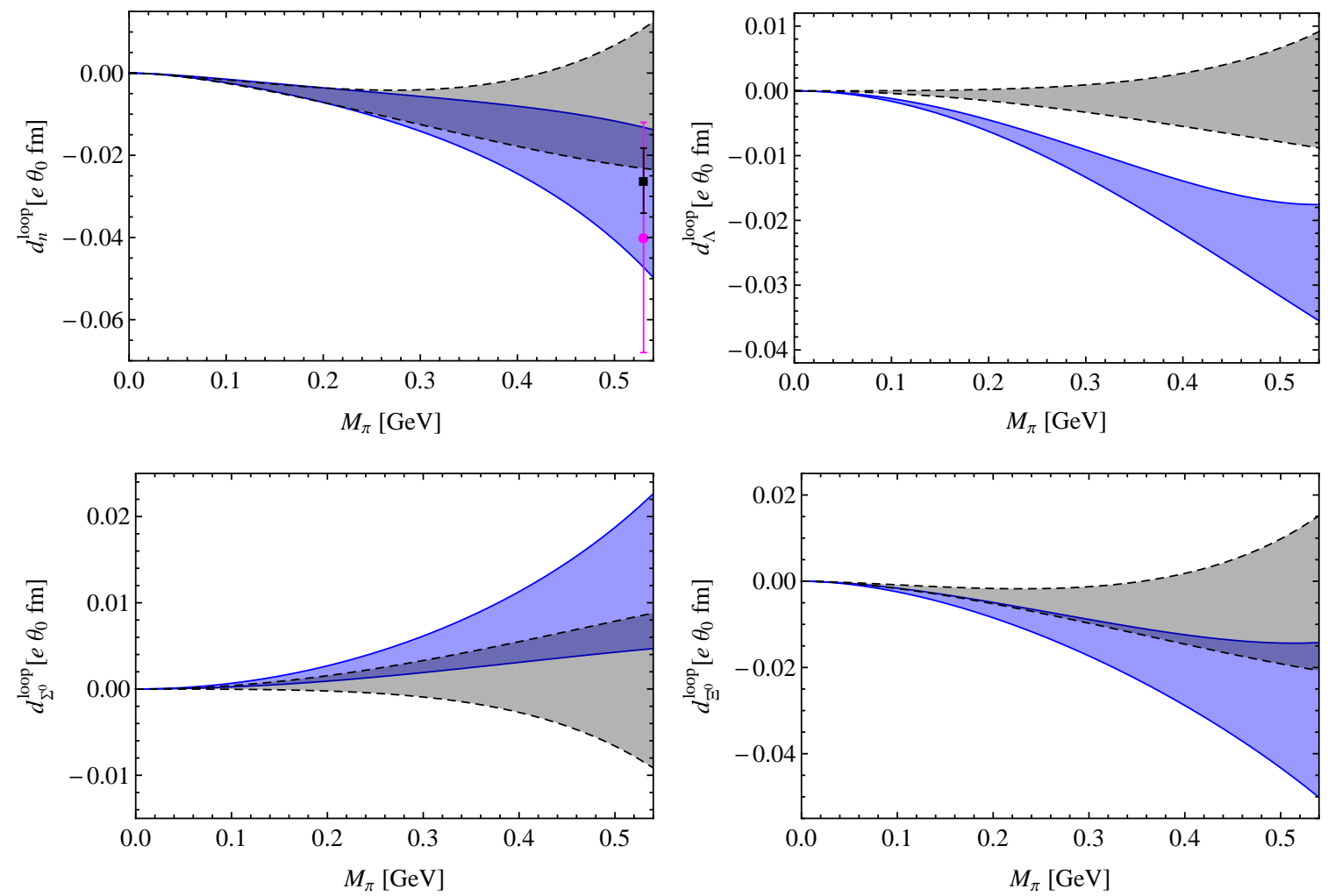

Figure 3. Loop contributions to the EDMs of the neutral baryons as a function of the pion mass. The bands, reflecting uncertainties by varying the renormalization scale between $\mu=M_{\rho}$ and $m_{\Xi}$, between solid and dashed boundaries are the NLO and the LO results, respectively. The filled circle and square with error bars are the lattice data from refs. [11] and [16], respectively.

we take $92.2 \mathrm{MeV}$ [39] for its value. From an analysis of the $\eta-\eta^{\prime}$ mixing in the framework of $\mathrm{U}(3)_{L} \times \mathrm{U}(3)_{R} \mathrm{CHPT}$, it was found that $V_{0}^{(2)}=-5 \times 10^{-4} \mathrm{GeV}^{4}$ and $V_{3}^{(1)}=3.5 \times 10^{-4} \mathrm{GeV}^{2}$ [40]. With these values, the neutron EDFF calculated at $\mu=1 \mathrm{GeV}$ is plotted in figure 2, where only 
the loop contribution is taken into account. One sees sizable NLO effects, especially for higher pion masses. One notices that the dependence on $q^{2}$ can be well approximated by a linear function, which means that a linear extrapolation from finite to vanishing $q^{2}$ can be used on the lattice. The same is true for the other baryons, as none of them shows a strong $q^{2}$-dependence.

At the physical pion mass, we get the loop contributions to the baryon EDMs in units of $10^{-16} e \theta_{0} \mathrm{~cm}$,

$$
\begin{array}{ll}
d_{n}^{\text {loop }}=-3.1 \pm 0.8, & d_{p}^{\text {loop }}=5.6 \pm 1.0-6.1(\beta \cdot \mathrm{GeV}), \\
d_{\Lambda}^{\text {loop }}=-2.6 \pm 0.4, & d_{\Sigma^{+}}^{\text {loop }}=3.8 \pm 1.0-4.8(\beta \cdot \mathrm{GeV}), \\
d_{\Sigma^{0}}^{\text {loop }}=0.8 \pm 0.4, & d_{\Sigma^{-}}^{\text {loop }}=-2.1 \pm 0.2+4.8(\beta \cdot \mathrm{GeV}), \\
d_{\Xi^{0}}^{\text {loop }}=-3.6 \pm 0.8, & d_{\Xi^{-}}^{\text {loop }}=-3.7 \pm 0.2+4.3(\beta \cdot \mathrm{GeV}),
\end{array}
$$

where the uncertainties are estimated by varying the scale $\mu$ between the masses of the $\rho$ and $\Xi$. If we replace the baryon masses in the $\beta$-term in the baryon EDFF expressions, eqs. (C.2), (C.4), (C.5) and (C.8), by the averaged baryon mass $m_{\text {ave }}=1151 \mathrm{MeV}$ as that in eq. (3.9), this term contributes $-5.0(\beta \cdot \mathrm{GeV})$ to the proton and $\Sigma^{+}$and $5.0(\beta \cdot \mathrm{GeV})$ to the $\Sigma^{-}$and $\Xi^{-}$. The difference reflects part of the higher order uncertainties. In the following, we will use the averaged baryon mass for the $\beta$-term and keep different masses for the other terms.

In order to compare with the results from lattice simulations, we should study the pion mass dependence of the pertinent quantities. We take the physical values for $F_{\pi}, M_{\eta_{8}, \eta_{0}}$ and the baryon masses, since their $M_{\pi}$-dependent effects contribute at higher orders. For the kaon mass, we use

$$
M_{K}^{2}=\stackrel{\circ}{M}_{K}^{2}+\frac{M_{\pi}^{2}}{2},
$$

where $\stackrel{\circ}{M}_{K}=484 \mathrm{MeV}$ is the kaon mass in the SU(2) chiral limit with vanishing up and down quark masses. The pion mass dependence of the EDMs of the neutral baryons is shown in figure 3, where the shaded bands between the solid and dashed boundaries are the NLO and LO results, respectively. It is clear that all the EDMs vanishes in the chiral limit. The physical reason is that the $\theta$-term can be rotated away if any of the quarks are massless, see, for instance, ref. [31], which ensures a vanishing value for the $\theta$-term induced EDM.

For comparison, the lattice data for the neutron at $M_{\pi}=530 \mathrm{MeV}$ calculated in refs. [11] are shown, which are calculated on $24^{3} \times 48$ lattice with lattice spacing $a \approx 0.11 \mathrm{fm}$. We also show the lattice data reported very recently in ref. [16] whose uncertainty is smaller. Both the LO and NLO loop results agree with the lattice data.

One notices that the NLO corrections for all the neutral hyperons are dramatic. For the $\Lambda$, from table 2 and eq. (3.3), one finds that the loops involving $\pi^{+}$and $\Sigma^{-}$cancel with those of $\pi^{-}$ and $\Sigma^{+}$exactly. Thus, the remaining LO contributions come from loops involving a kaon. They are small as can be seen from

$$
1+\ln \frac{M_{\pi}^{2}}{\mu^{2}}=-2.96, \quad 1+\ln \frac{M_{K}^{2}}{\mu^{2}}=-0.40,
$$

with $\mu=1 \mathrm{GeV}$. Therefore, the LO result for the $\Lambda$ EDM is close to zero (as already pointed out in ref. [22]). The same happens for the $\Sigma^{0}$. 

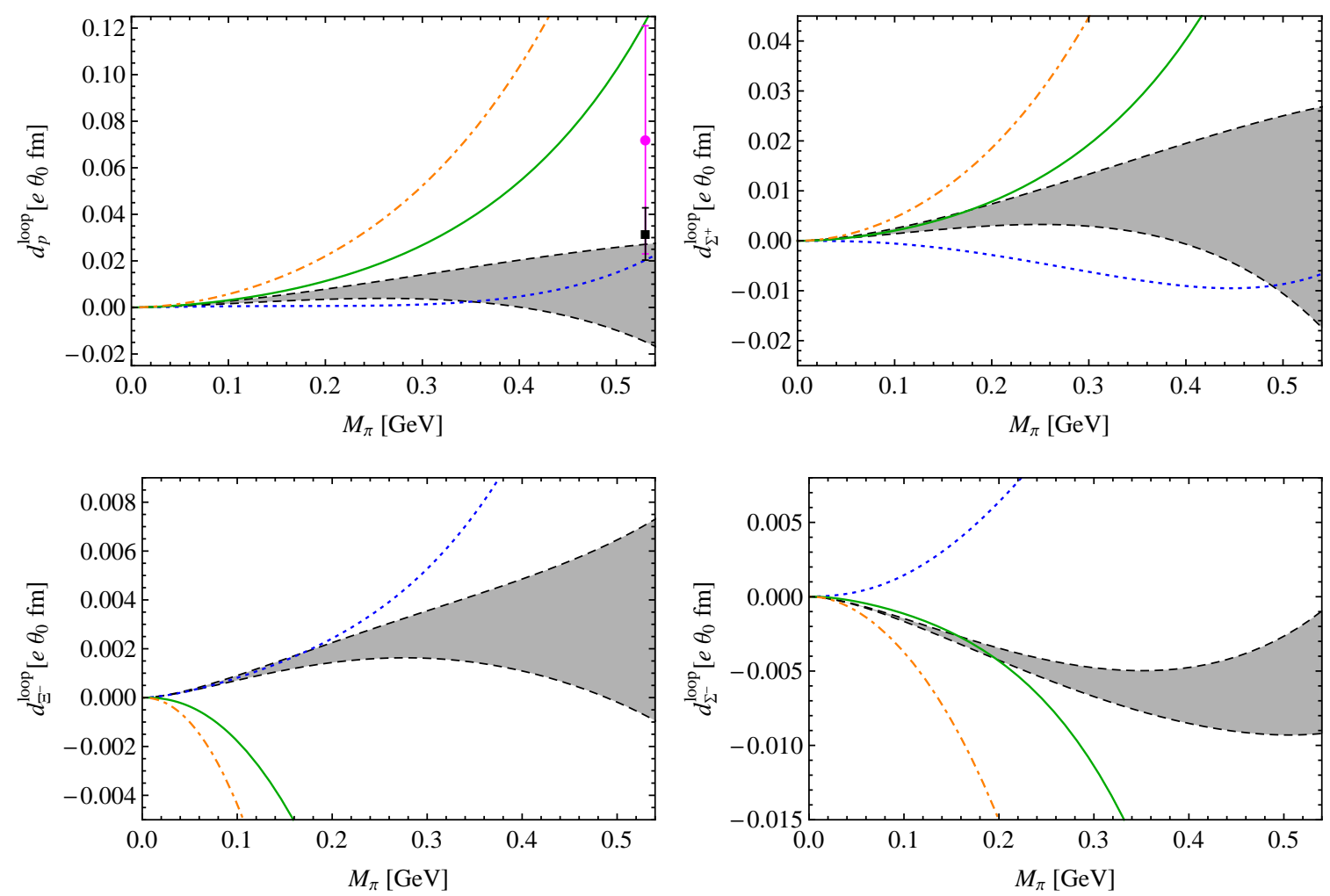

Figure 4. Loop contributions to the EDMs of the charged baryons as a function of the pion mass. The bands, reflecting uncertainties of the LO contributions by varying the renormalization scale between $\mu=m_{\rho}$ and $m_{\Xi}$. The filled circle and square with error bars are the lattice data from refs. [11] and [16], respectively. The solid, dotted and dot-dashed lines represent the NLO results evaluated at $\mu=1 \mathrm{GeV}$ with $\beta=0,1$ and $-1 \mathrm{GeV}^{-1}$, respectively.

The results for the charged baryons are shown in figure 4, where the bands are the LO loop results. We choose three different values for the unknown combination $\beta$ to illustrate the NLO effects. The results are quite sensitive to the numerical value of $\beta$, especially for the $\Xi^{-}$and $\Sigma^{-}$.

\subsection{A first determination of the LECs from lattice results}

We may use the neutron and the proton EDMs at unphysical quark masses to determine the two combination of parameters $w_{a}(\mu)$ and $w_{b}(\mu)$. Using the lattice data at $M_{\pi}=530 \mathrm{MeV}[16]^{3}$ for the neutron EDM, one may determine the counterterm combination $w_{a}(\mu)$

$$
w_{a}(1 \mathrm{GeV})=(-0.01 \pm 0.02) \mathrm{GeV}^{-1},
$$

where the uncertainty merely reflects the uncertainty in the lattice calculations. Using the data for the proton, we get

$$
w_{b}(1 \mathrm{GeV})=(-0.40 \pm 0.05) \mathrm{GeV}^{-1} .
$$

\footnotetext{
${ }^{3}$ As this pion mass value is at the edge of the range of applicability of our approach, lattice data at lower pion mass are urgently called for for a more reliable determination of the LECs.
} 


\begin{tabular}{|l|cccccccc|}
\hline Baryon & $p$ & $n$ & $\Sigma^{+}$ & $\Sigma^{0}$ & $\Sigma^{-}$ & $\Lambda$ & $\Xi^{0}$ & $\Xi^{-}$ \\
\hline Combination & $-\left(w_{a}+w_{b}\right)$ & $2 w_{a}$ & $-\left(w_{a}+w_{b}\right)$ & $-w_{a}$ & $w_{b}-w_{a}$ & $w_{a}$ & $2 w_{a}$ & $w_{b}-w_{a}$ \\
\hline
\end{tabular}

Table 4. Combinations of the unknown parameters appearing in the EDFFs of the baryons up to NLO.

With these determinations, the EDMs at the physical pion mass can be predicted including both the tree and the loop contributions. The ones for the neutron and proton in units of $10^{-16} e \theta_{0} \mathrm{~cm}$ are

$$
d_{n}=-2.9 \pm 0.4 \pm 0.8, \quad d_{p}=1.1 \pm 0.5 \pm 1.0 .
$$

Here, the first uncertainty reflects the uncertainty in the determination of $w_{a}(1 \mathrm{GeV})$ and $w_{b}(1 \mathrm{GeV})$, and the second one corresponds to varying the scale $\mu$ between the $\rho$-meson mass and $m_{\Xi}$. Comparing with the loop results given in eq. (3.10), one sees that the neutron EDM at the physical pion mass is dominated by the loops. For the proton, the loop contributions have similar size as, albeit slightly larger than, the tree level terms. Combining these two errors, we have $d_{n}=-2.9 \pm 0.9$ and $d_{p}=d_{p}=1.1 \pm 1.1$ (in canonical units). Using the experimental upper limit of the neutron EDM, $\left|d_{n}\right|<2.9 \times 10^{-26} e \mathrm{~cm}[1]$, the $\theta_{0}$ angle is constrained to be

$$
\left|\theta_{0}\right| \lesssim 1.5 \times 10^{-10}
$$

Similarly, we can predict the hyperon EDMs (again in units of $10^{-16} e \theta_{0} \mathrm{~cm}$ )

$$
\begin{array}{rlrl}
d_{\Lambda} & =-2.5 \pm 0.2 \pm 0.4, & & d_{\Sigma^{+}}=-0.7 \pm 0.5 \pm 1.0 \\
d_{\Sigma^{0}}=0.7 \pm 0.2 \pm 0.4, & & d_{\Sigma^{-}}=2.2 \pm 0.5 \pm 0.2 \\
d_{\Xi^{0}}=-3.4 \pm 0.4 \pm 0.8, & & d_{\Xi^{-}}=0.6 \pm 0.5 \pm 0.2 .
\end{array}
$$

\subsection{Counterterm-free relations}

In this section, we will derive relations that are free of the unknown LECs up to NLO. These relations can e.g. serve as checks of lattice simulations for varying quark masses. In fact, due the $\mathrm{SU}(3)$ flavor symmetry, there exists an exact relation among the EDFFs of the $\Sigma$ hyperons. From table 2, one deduces that the loops involving a pion or an $\eta_{8(0)}$ for the $\Sigma^{+}$cancel with those of the $\Sigma^{-}$. As mentioned before, the pionic loops of the $\Sigma^{0}$ cancel with each other. Furthermore, there are also cancellations for the kaonic loops among the $\Sigma$ hyperons. These cancellations occur when isospin is a good symmetry. Therefore, in the isospin symmetric case, which is considered throughout, one has

$$
F_{3, \Sigma^{+}}+F_{3, \Sigma^{-}}-2 F_{3, \Sigma^{0}}=\mathcal{O}\left(\delta^{4}\right) .
$$

This relation is exact up to NLO including the tree-level contributions. Similarly, there is another relation which is exact only at $\mathrm{LO}$

$$
F_{3, \Sigma^{0}}+F_{3, \Lambda}=\mathcal{O}\left(\delta^{3}\right)
$$

since the LO loop contributions are independent of the baryon mass, and they cancel each other in the sum. Moreover, the above combination is also independent of $\beta$ which combines the unknown 

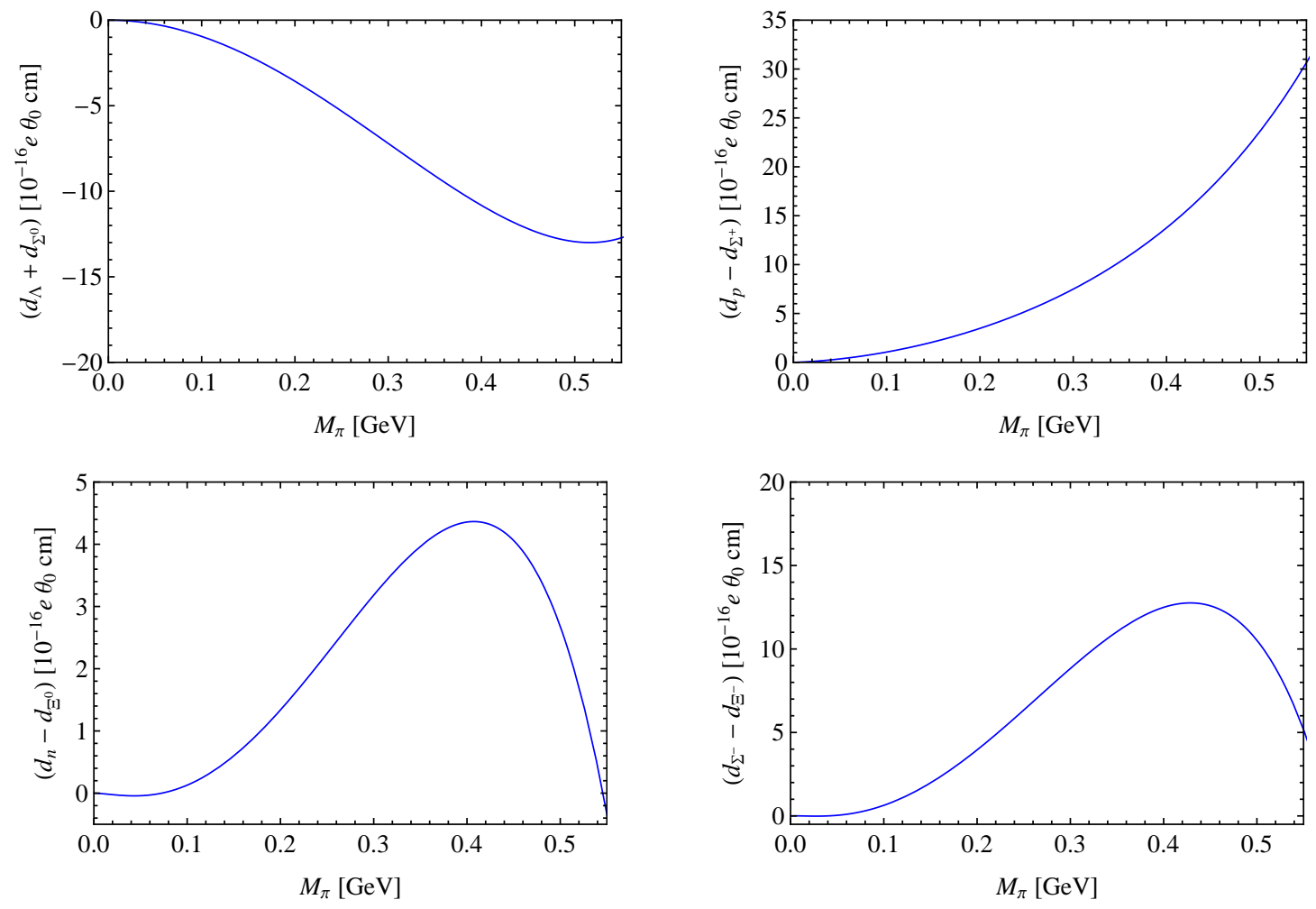

Figure 5. Counterterm-free combinations of the baryon EDMs as a function of the pion mass.

parameters $w_{0}$ and $w_{10}^{\prime}$, so that it can be calculated parameter-free. Taking $q^{2}=0$, the $\mathcal{O}\left(\delta^{3}\right)$ expression is rather simple

$$
d_{\Sigma^{0}}+d_{\Lambda}=-\frac{16 e V_{0}^{(2)} \bar{\theta}_{0}}{3 \pi F_{\pi}^{4} M_{K}}\left(M_{K}^{2}-M_{\pi}^{2}\right)\left(F b_{D}^{2}+2 D b_{D} b_{F}+3 F b_{F}^{2}\right)+\mathcal{O}\left(\delta^{4}\right) .
$$

In fact, there are more combinations free of the counterterms. As mentioned before, up to NLO, there are only two combinations of unknown constants, $w_{a}(\mu)$ and $w_{b}(\mu)$, in the expressions of the EDFFs of the octet baryons defined in eqs. (3.8) and (3.9). It is easy to find combinations free of any unknown parameters up to $\mathcal{O}\left(\delta^{3}\right)$ utilizing the results collected in table 4 . In addition to the two in eqs. (3.17) and (3.19), they include

$$
F_{3, p}-F_{3, \Sigma^{+}}, \quad F_{3, n}-F_{3, \Xi^{0}}, \quad F_{3, \Sigma^{-}}-F_{3, \Xi^{-}} .
$$

Because the counterterms have been cancelled out, these combinations are also finite and hence independent of the scale $\mu$. However, different from eq. (3.19), their LO loop contributions do not vanish. The expression for the $F_{3, n}-F_{3, \Xi^{0}}$ at $q^{2}=0$ reads

$$
\begin{aligned}
d_{n}-d_{\Xi^{0}}= & \frac{e V_{0}^{(2)} \bar{\theta}_{0}}{\pi^{2} F_{\pi}^{4}}\left[\left(D b_{D}+F b_{F}\right)\left(2 \ln \frac{M_{K}^{2}}{M_{\pi}^{2}}+\pi \frac{M_{\pi}-M_{K}}{m_{\text {ave }}}\right)\right. \\
& \left.+\frac{8 \pi}{M_{K}}\left(M_{K}^{2}-M_{\pi}^{2}\right)\left(D b_{D}^{2}+2 F b_{D} b_{F}+D b_{F}^{2}\right)\right]+\mathcal{O}\left(\delta^{4}\right) .
\end{aligned}
$$


The relations for the charged baryons are more complicated, and are not shown here (they can be obtained from the expressions given in appendix C). The pion mass dependence of the four combinations in eqs. (3.18) and (3.20) are shown in figure 5. Their values at the physical pion mass in units of $10^{-16} e \theta_{0} \mathrm{~cm}$ are

$$
\begin{array}{ll}
d_{\Sigma^{0}}+d_{\Lambda}=-1.8, & d_{p}-d_{\Sigma^{+}}=1.8 \\
d_{n}-d_{\Xi^{0}}=0.5, & d_{\Sigma^{-}}-d_{\Xi^{-}}=1.6 .
\end{array}
$$

Note that although the first combination starts from one order higher than the others, their numerical values are of similar order of magnitude.

\section{Finite-volume corrections in the $p$-regime}

On the lattice, calculations are performed in a finite volume. As a result, the continuum momentum spectrum becomes quantized. Taking periodic boundary condition for all three spatial dimensions forms a torus. If the volume is $L^{3}$, the momentum takes values of $2 \pi \vec{n} / L$, with $\vec{n}$ a three-dimensional vector of integers. One easily sees that any integral over the spatial components of momentum in the infinite volume should be replaced by a summation for the momentum modes. For instance, the two-point scalar loop integral needs to be changed as follows,

$$
i \int \frac{d^{4} k}{(2 \pi)^{4}} \frac{1}{\left(k^{2}-m_{1}^{2}\right)\left[(k+q)^{2}-m_{2}^{2}\right]} \rightarrow \frac{i}{L^{3}} \sum_{\vec{n}} \int \frac{d k^{0}}{2 \pi} \frac{1}{\left(k^{2}-m_{1}^{2}\right)\left[(k+q)^{2}-m_{2}^{2}\right]} .
$$

The finite volume corrections to a quantity $\mathcal{Q}$ is defined as the difference of $\mathcal{Q}$ evaluated in a finite and infinite volume,

$$
\delta_{L}[\mathcal{Q}]=\mathcal{Q}(L)-\mathcal{Q}(\infty)
$$

As we already mentioned, at chiral limit the baryon EDMs should vanish. However, the current lattice data at rather large pion masses do not show a decreasing behavior yet [10,11]. Thus, results at smaller pion masses are necessary. However, in the section, we will show that the finite volume corrections at small pion masses to most of the baryon EDMs are quite large, and it is necessary to include the NLO contributions. The decomposition of the matrix element of the electromagnetic current in the form of eq. (3.1) assumes Lorentz invariance. However, on discretized lattice, Lorentz invariance in infinite volume is lost. Furthermore, the external momentum is also quantized in a finite volume. These effects should be taken into account when calculating the finite volume corrections to the form factors [41, 42]. Since we are not aiming at a very precise calculation of the finite volume effects, we will simply assume Lorentz invariance in the following.

As shown in ref. [43], one can use the same LECs as in the infinite volume, and the finite volume corrections come only from loops. This may be understood easily, since the finite volume effects are long-distance physics, while the LECs reflect the short-distance physics. For the same reason, the finite volume corrections should not depend on the choice of method to regularize the ultraviolet divergence. This means that one may change the upper bound of the Feynman parameter integration in eqs. (D.5) and (D.7) in the infrared regularization from $\infty$ to 1 as already noticed in, for instance, ref. [44]. In the $p$-regime, where $M_{\pi} L \gg 1$ [43, 45], the finite volume corrections are exponentially suppressed. 

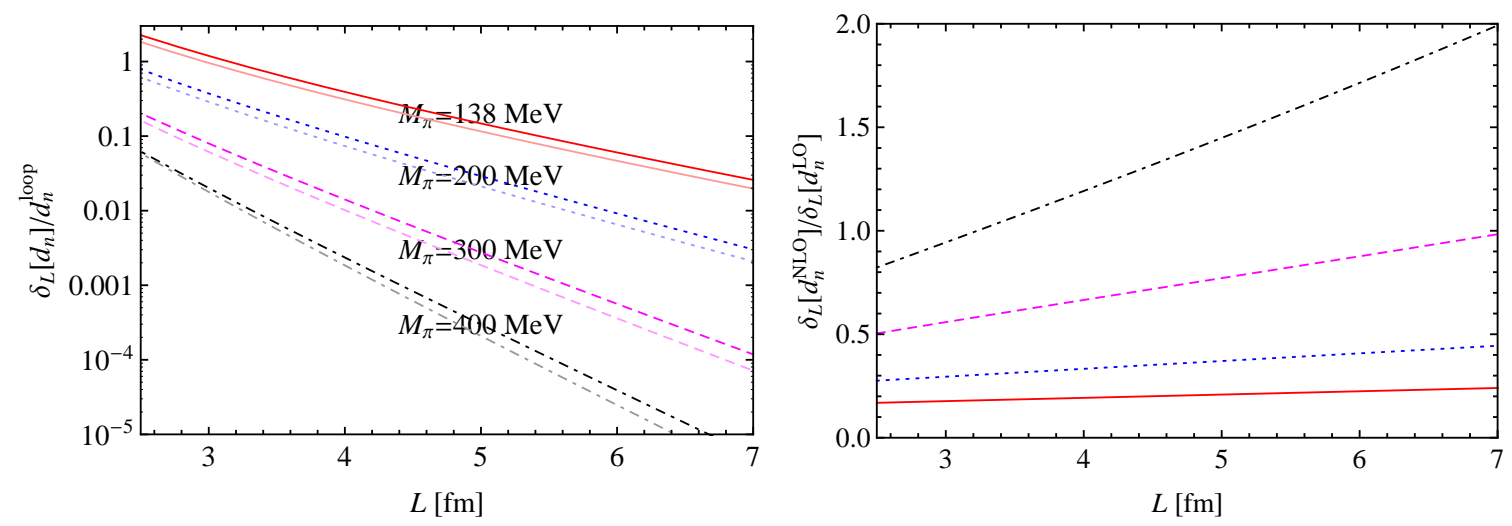

Figure 6. Left: The ratios of the finite volume corrections to the loop contributions in the infinite volume for the neutron EDM as a function of $L$. Right: The ratios of the finite volume corrections at NLO (LO not included) to those at LO. The solid, dotted, dashed and dot-dashed lines are for the pion mass being physical, $200 \mathrm{MeV}, 300 \mathrm{MeV}$ and $400 \mathrm{MeV}$, respectively. In the left panel, for each value of the pion mass, two lines are plotted with the upper and lower ones representing the NLO and LO results, respectively.

In ref. [29], finite volume correction to the neutron EDM was calculated at LO in SU(2) heavy baryon CHPT. The resulting expression is given by [29]

$$
\delta_{L}\left[d_{n}^{\mathrm{LO}}\right]=\frac{g_{A} \bar{\alpha} e \theta_{0}}{2 \pi^{2} F_{\pi}^{2}} \frac{m_{u} m_{d}}{m_{u}+m_{d}} \sum_{\vec{n} \neq 0} K_{0}\left(L M_{\pi}|\vec{n}|\right),
$$

where $\bar{\alpha}$ is a coefficient of the CP-violating $N N \pi$ vertex. ${ }^{4}$ From eqs. (C.1) and (D.4), our result for the finite volume correction to the neutron EDM at LO is

$\delta_{L}\left[d_{n}^{\mathrm{LO}}\right]=\frac{M_{\pi}^{2} e \theta_{0}}{4 \pi^{2} F_{\pi}^{2}} \sum_{\vec{n} \neq 0}\left[(D+F)\left(b_{D}+b_{F}\right) K_{0}\left(L M_{\pi}|\vec{n}|\right)-(D-F)\left(b_{D}-b_{F}\right) K_{0}\left(L M_{K}|\vec{n}|\right)\right]$,

where we have used [22]

$$
\bar{\theta}_{0} \simeq \frac{F_{\pi}^{2} M_{\pi}^{2}}{8 V_{0}^{(2)}} \theta_{0}
$$

which may be obtained from eq. (2.14) considering $M_{\pi} \ll M_{K}$. Comparing the SU(2) part with eq. (4.3), we can identify

$$
\bar{\alpha}=\frac{B_{0}}{2}\left(b_{D}+b_{F}\right) .
$$

The left panel of figure 6 shows the ratios of the finite volume corrections to the loop contributions in the infinite volume for the neutron EDM as a function of $L$. The results are obtained with $\mu=1 \mathrm{GeV}$. In order to show the impact of the NLO corrections more clearly, we plot the ratios of the finite volume corrections at NLO (LO not included) to the LO in the right panel of figure 6. One sees that at the physical pion mass the NLO contributions increase the LO finite volume corrections by about $20 \%$. This correction is much larger for a pion mass of $400 \mathrm{MeV}$. At this point, one might worry about the convergence. In fact, the neutron EDM in the infinite volume

\footnotetext{
${ }^{4} \bar{\alpha}$ is the $\alpha$ used in ref. [29].
} 

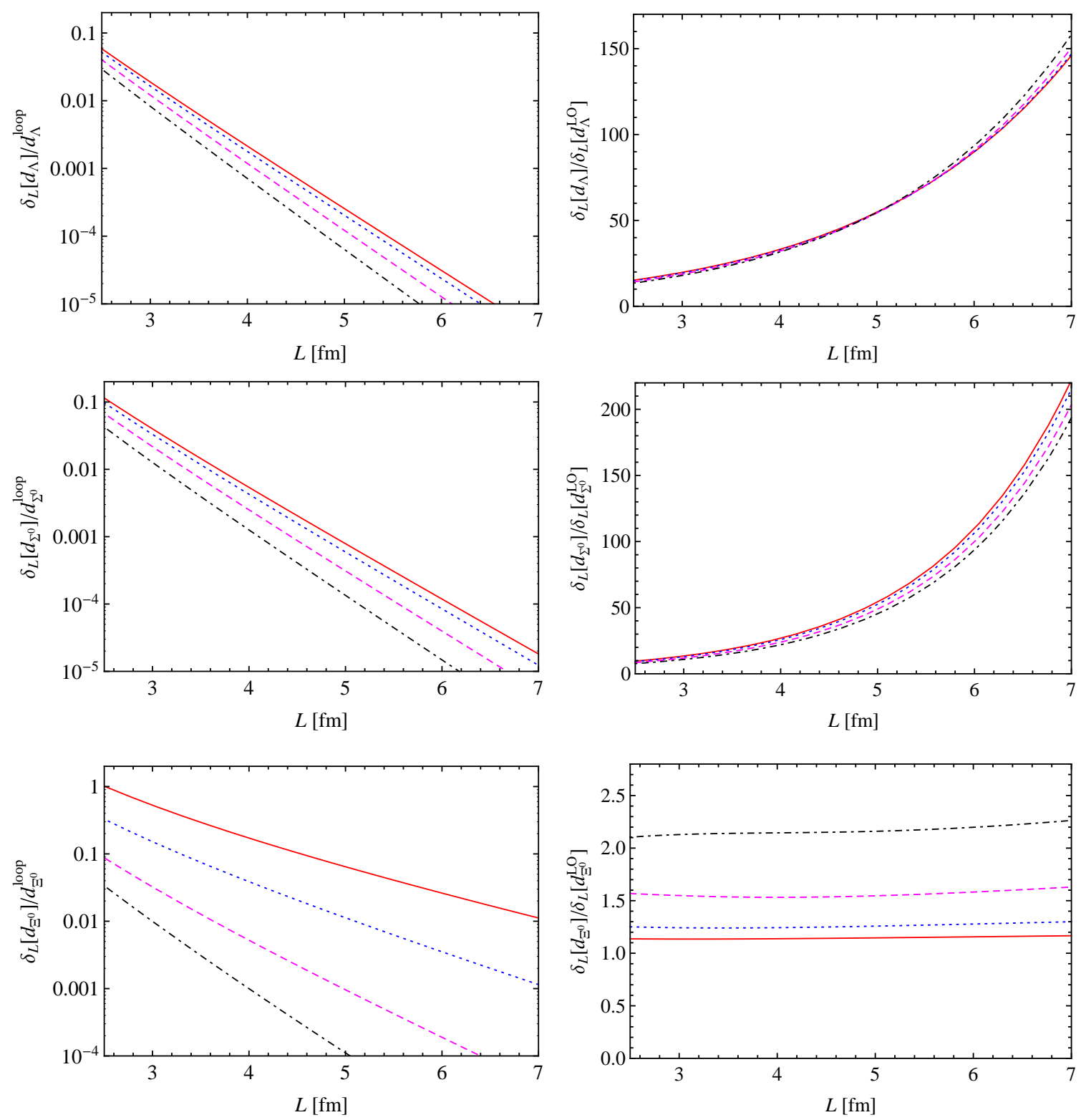

Figure 7. Left: The ratios of the finite volume corrections to the loop contributions in the infinite volume for the EDMs of neutral hyperons as a function of $L$. Right: The ratios of the finite volume corrections of NLO to those of LO. The solid, dotted, dashed and dot-dashed lines are for the pion mass being $138 \mathrm{Nev}$ (physical value), $200 \mathrm{MeV}, 300 \mathrm{MeV}$ and $400 \mathrm{MeV}$, respectively.

has a nice convergence property, as can be seen from the left panel of figure 3. Moreover, one can show that the ratio tends to increase with increasing $L$. Although both the LO and NLO finite volume corrections decrease exponentially, the LO one decreases faster. Using eqs. (E.2) and (E.4) from appendix E, we get in the limit $L \rightarrow \infty$,

$$
\frac{\delta_{L}\left[d_{n}^{\mathrm{NLO}}\right]}{\delta_{L}\left[d_{n}^{\mathrm{LO}}\right]} \sim \sqrt{\frac{\pi}{2}} \frac{M_{\pi}}{m_{N}} \sqrt{L M_{\pi}} \exp \left(\frac{L M_{\pi}^{3}}{8 m_{N}^{2}}\right) .
$$

The finite volume corrections to the neutral hyperons are shown in figure 7. It is obvious that the 

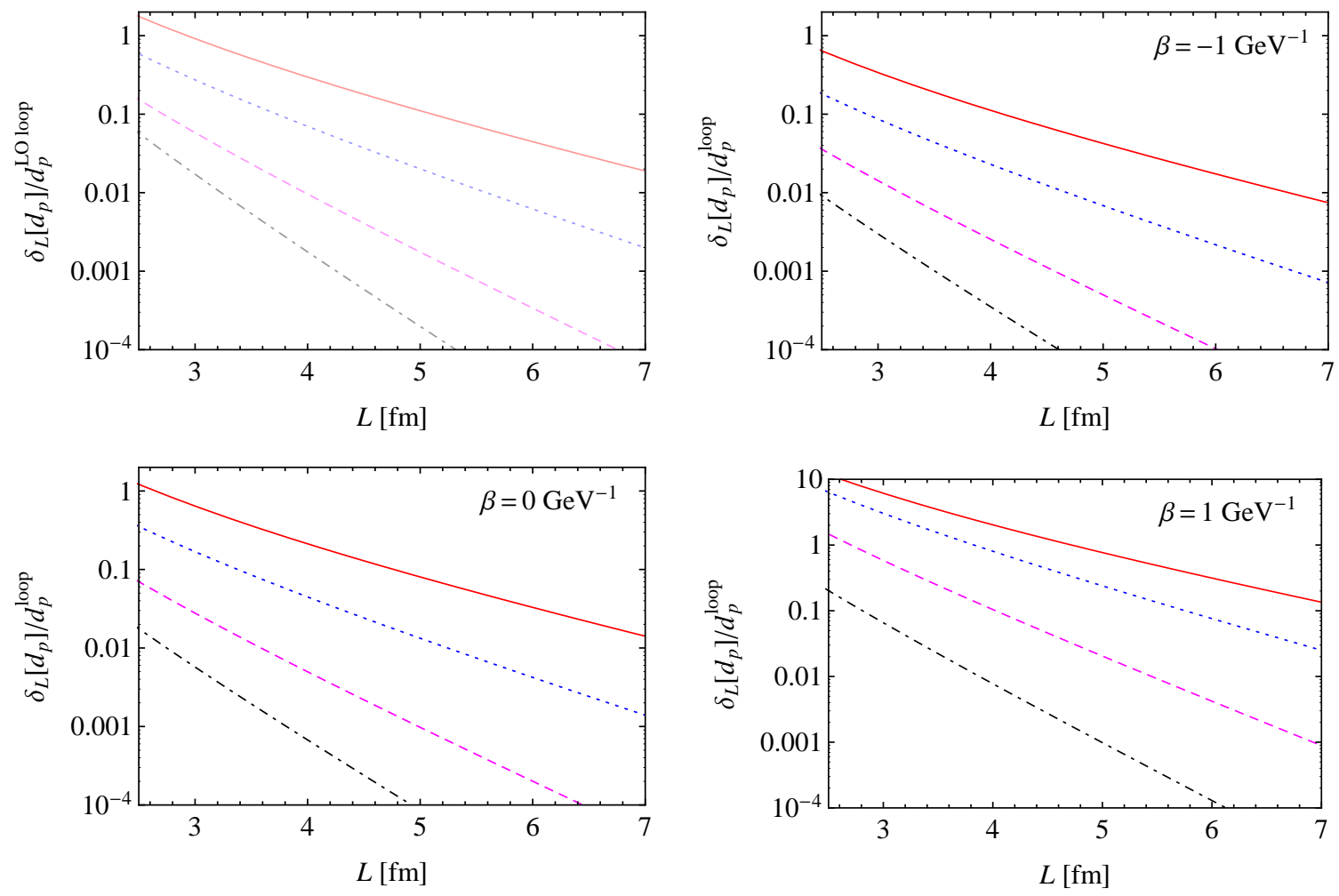

Figure 8. The ratio of the finite volume corrections to the loop contributions in the infinite volume for the proton EDM as a function of $L$. The first graph presents the LO results, and the others are the NLO results for different choices of $\beta$. The solid, dotted, dashed and dot-dashed lines are for the pion mass of $138 \mathrm{MeV}$, $200 \mathrm{MeV}, 300 \mathrm{MeV}$ and $400 \mathrm{MeV}$, respectively.

effects are very small for the $\Lambda$ and $\Sigma^{0}$. The reason is that the pionic loops with positively and negatively charged pions cancel each other exactly for these two baryons. This also makes the NLO corrections much larger than the $\mathrm{LO}$ ones at $\mu=1 \mathrm{GeV}$, consistent with the infinite volume results shown in figure 3. The situation for the $\Xi^{0}$ is similar to case of the neutron.

Although the parameter combination $w_{b}(\mu)$ can be determined from the lattice data of the proton EDM, $\beta$ is still unknown since it always appear together with the LECs $w_{14}$ and $w_{14}^{\prime r}(\mu)$. However, the finite volume corrections for the charged baryons depend on $\beta$, but not on $w_{14}$ and $w_{14}^{\prime r}(\mu)$. It is therefore possible to extract the value of $\beta$ from the volume effects of the baryon EDMs. In figure 8, we show the ratios of the finite volume corrections to the loop contributions in infinite volume to the proton EDM for different chosen values of $\beta$. At this point, we want to emphasize that the EDMs of all the charged baryons depend on the same combination of LECs, i.e. $\beta$. Once it is determined from one baryon, it can be used for predicting the finite volume corrections to the others.

\section{Summary}

In this paper, we extended the previous calculation [27] of the nucleon EDFFs and EDMs to the ground state baryon octet up to NLO in the framework of $\mathrm{U}(3)_{L} \times \mathrm{U}(3)_{R}$ chiral perturbation theory. 
Our main findings can be summarized as follows:

1) We have shown that the complete one-loop expressions for the baryon EDFFs and EDMs depend on two combinations of unknown LECs only, $w_{a}$ and $w_{b}$, cf. eqs.(3.8,3.9). In case of the charged baryons, the combination $w_{b}(\mu)$ combines two LECs from the tree graphs and one LEC that appears only in loops.

2) We have shown that the NLO corrections are large for the neutral hyperons $\Lambda$ and $\Sigma^{0}$. This is due to a suppression of the LO contributions based on exact cancellations between loops of positively and negatively charged pions. For the charged baryons, we find a strong sensitivity to the LEC combination $w_{b}$.

3) We have derived a set of relations between various EDMs that are free of unknown LECs. These can be useful for future lattice simulations of baryon EDMs.

4) Based on recent lattice results for the neutron and proton EDMs at $M_{\pi}=530 \mathrm{MeV}$, we could pin down the two LEC combinations $w_{a}$ and $w_{b}$. Based on this, we can predict the baryon EDMs at the physical pion mass. In particular, we find $d_{n}=-2.9 \pm 0.9$ and $d_{p}=1.1 \pm 1.1$ in units of $10^{-16} \theta_{0} e \mathrm{~cm}$.

5) The finite volume corrections to the baryon EDMs in the p-regime are also studied. Because the loops contribute from LO, it is found that the finite volume corrections are huge for all the baryon EDMs except for the $\Lambda$ and $\Sigma^{0}$ for the pion mass close to its physical value. For the neutron, the finite volume correction is about $10 \%$ at $M_{\pi} L=4$.

Note that the calculation of the finite volume corrections in our paper assumes Lorentz invariance, and additional subtleties in the presence of external electromagnetic fields, see [46], are not taken into account. For a precise calculation of the finite volume corrections of the baryon EDMs, separate analyses need to be done for the lattice calculations using the form factor method, the spectrum method with external field and methods with the twisted boundary conditions.

More lattice results of the nucleon EDMs are expected to come out soon. Calculations of the hyperons are welcome based on our analysis. With these upcoming lattice data, one can determine the LECs more reliably, and a next-to-next-to-leading order calculation would be feasible and desirable in view of the bad convergence for some baryons. Furthermore, it would be interesting to see how the LEC-free relations get modified at higher orders.

\section{Acknowledgments}

We are grateful to E. Shintani for providing us with lattice data before publication. We acknowledge discussions with T. Izubichi and G. Schierholz. We also thank B. C. Tiburzi for very useful comments on the finite volume corrections. This work is supported in part by the DFG and the NSFC through funds provided to the Sino-German CRC 110 "Symmetries and the Emergence of Structure in QCD" and the EU I3HP "Study of Strongly Interacting Matter" under the Seventh Framework Program of the EU. U.-G. M. also thanks the BMBF for support (Grant No. 06BN7008). F.-K. G. acknowledges partial support from the NSFC (Grant No. 11165005). 


\begin{tabular}{|l|cccc|}
\hline Baryons & $N$ & $\Sigma$ & $\Lambda$ & $\Xi$ \\
\hline$b_{\pi}$ & $b_{F}$ & $b_{D}$ & $-b_{D} / 3$ & $-b_{F}$ \\
$b_{K}$ & $b_{D}-b_{F}$ & 0 & $4 b_{D} / 3$ & $b_{D}+b_{F}$ \\
\hline
\end{tabular}

Table 5. Coefficients $b_{\pi, K}$ in the NLO baryon mass formula.

\section{A Baryon masses up to NLO}

From eq. (2.16), it is easy to obtain the baryon masses up to $\mathcal{O}\left(\delta^{2}\right)$, which may be written as

$$
m_{B}=\stackrel{\circ}{m}-2 b_{0}\left(M_{\pi}^{2}+2 M_{K}^{2}\right)-4\left(b_{\pi} M_{\pi}^{2}+b_{K} M_{K}^{2}\right)+\mathcal{O}\left(\delta^{3}\right),
$$

where we have expressed the quark masses in terms of the meson masses at the lowest order, and $b_{\pi, K}$ are listed in table 5 .

\section{B Loop integrals in infrared regularization}

Loop integrals in the infinite volume used in this paper are collected in this appendix. Let us denote the masses of the light meson and baryon in the loop by $M$ and $m$, respectively. The momentum of the external current is $q^{\mu}$, and the momenta of the external baryons are denoted by $p^{\mu}$ and $p^{\prime \mu}=p^{\mu}+q^{\mu}$, respectively. We further define

$$
L=\frac{\mu^{d-4}}{(4 \pi)^{2}}\left\{\frac{1}{d-4}-\frac{1}{2}\left[\ln (4 \pi)+\Gamma^{\prime}(1)+1\right]\right\}
$$

which contains the divergence at the spacetime dimension $d=4$.

The 2-point mesonic-loop function when the two mesons have the same mass reads as

$$
\begin{aligned}
J_{M M}\left(q^{2}\right) & =i \int \frac{d^{d} k}{(2 \pi)^{d}} \frac{1}{\left(k^{2}-M^{2}+i \epsilon\right)\left[(k+q)^{2}-M^{2}+i \epsilon\right]} \\
& =2 L+\frac{1}{16 \pi^{2}}\left(\ln \frac{M^{2}}{\mu^{2}}-1-\sigma \ln \frac{\sigma-1}{\sigma+1}\right),
\end{aligned}
$$

where $\sigma=\sqrt{1-4 M^{2} / q^{2}}$.

When there is a baryon in the loop, the loop evaluated in the normal dimensional regularization spoils power counting [47]. The power counting can be restored in the heavy baryon formalism of baryon CHPT [48, 49]. There are also covariant formalisms, see ref. [50] for a recent review.

Here, the infrared regularization $[36,51]$ will be used. In the infrared regularization, the integral is separated into an infrared regular piece and an infrared singular piece. The infrared regular piece can be expanded analytically in the chiral expansion, and absorbed into the counterterms. Only the infrared singular part of the loop integrals will be shown in the following.

The two-point scalar loop integral involving one meson and one baryon is

$$
\begin{aligned}
J_{M m}\left(p^{2}\right) & =i \int \frac{d^{d} k}{(2 \pi)^{d}} \frac{1}{\left(k^{2}-M^{2}+i \epsilon\right)\left[(p-k)^{2}-m^{2}+i \epsilon\right]} \\
& =\frac{\omega}{p^{2}} L+\frac{1}{32 \pi^{2} p^{2}}\left[\omega\left(\ln \frac{M^{2}}{\mu^{2}}-1\right)+2 \sqrt{\lambda\left(p^{2}, m^{2}, M^{2}\right)} \arccos \left(-\frac{\omega}{2 \tilde{p} m}\right)\right]
\end{aligned}
$$


where $\omega=p^{2}-m^{2}+M^{2}, \tilde{p}=\sqrt{p^{2}}$, and $\lambda(x, y, z)=x^{2}+y^{2}+z^{2}-2(x y+y z+x z)$ is the Källén function. Noting that the external baryons are on shell, $p^{2}$ is given by the mass squared of the external baryon. Thus, with the baryon mass expressions in appendix A, one has $p^{2}=m^{2}+\mathcal{O}\left(\delta^{2}\right)$ and $\omega=\mathcal{O}\left(\delta^{2}\right)$. Expanding the arccos function, we get

$$
J_{M m}\left(p^{2}\right)=\frac{M}{16 \pi \tilde{p}}+\frac{\omega}{p^{2}}\left[L+\frac{1}{32 \pi^{2}}\left(1+\ln \frac{M^{2}}{\mu^{2}}\right)\right]+\mathcal{O}\left(\delta^{3}\right),
$$

where the first and second term are of $\mathcal{O}(\delta)$ and $\mathcal{O}\left(\delta^{2}\right)$, respectively.

The three-point loop integral with two mesons and one baryon can be written as

$$
\begin{aligned}
J_{M M m}\left(q^{2}, p^{2}\right) & =i \int \frac{d^{d} k}{(2 \pi)^{d}} \frac{1}{\left(k^{2}-M^{2}+i \epsilon\right)\left[(k+q)^{2}-M^{2}+i \epsilon\right]\left[(p-k)^{2}-m^{2}+i \epsilon\right]} \\
& =\frac{\partial}{\partial M^{2}} \int_{0}^{1} d x J_{\bar{M} m}\left(\bar{p}^{2}\right),
\end{aligned}
$$

where $\bar{M}^{2}=M^{2}+x(x-1) q^{2}, \bar{p}^{\mu}=p^{\mu}+x q^{\mu}$. In the following, $p^{2}=p^{2}$ will always be assumed, then one has $\bar{p}^{2}=p^{2}+x(x-1) q^{2}$. The leading chiral order of this integral is $\mathcal{O}\left(\delta^{-1}\right)$. Keeping terms up to $\mathcal{O}\left(\delta^{0}\right)$, the analytic expression for the infrared singular part is

$$
\begin{aligned}
J_{M M m}\left(q^{2}, p^{2}\right)= & \frac{1}{16 \pi \tilde{p} \sqrt{-q^{2}}} \arctan \frac{\sqrt{-q^{2}}}{2 M} \\
& +\left[\frac{L}{p^{2}}+\frac{1}{32 \pi^{2} p^{2}}\left(1+\ln \frac{M^{2}}{\mu^{2}}+\frac{2 \omega-q^{2}}{q^{2} \sigma} \ln \frac{\sigma-1}{\sigma+1}\right)\right]+\mathcal{O}(\delta),
\end{aligned}
$$

where the first term is of order $\mathcal{O}\left(\delta^{-1}\right)$, and the terms in the square brackets are of order $\mathcal{O}\left(\delta^{0}\right)$.

For completeness, we also give the expression for the loop with one meson and two baryons, though not used in our calculations. Similar to $J_{M M m}\left(q^{2}, p^{2}\right)$, this loop can also be worked out from the two-point loop with one meson and one baryon,

$$
\begin{aligned}
J_{M m m}\left(p^{2}, p^{\prime 2}\right) & =i \int \frac{d^{d} k}{(2 \pi)^{d}} \frac{1}{\left(k^{2}-M^{2}+i \epsilon\right)\left[(p-k)^{2}-m^{2}+i \epsilon\right]\left[\left(p^{\prime}-k\right)^{2}-m^{2}+i \epsilon\right]} \\
& =\frac{\partial}{\partial m^{2}} \int_{0}^{1} d x J_{M \bar{m}}\left(\bar{p}^{2}\right),
\end{aligned}
$$

where $\bar{m}^{2}=m^{2}+x(x-1) q^{2}$. The analytic expression up to NLO is

$$
J_{M m m}\left(p^{2}, p^{2}\right)=-\left[\frac{L}{p^{2}}+\frac{1}{32 \pi^{2} p^{2}}\left(1+\ln \frac{M^{2}}{\mu^{2}}\right)\right]+\frac{\omega}{64 \pi p^{2} m M}+\mathcal{O}\left(\delta^{2}\right),
$$

where the terms in the square brackets are at LO, and the second term is at NLO. Up to NLO, this loop does not depend on the momentum transfer $q^{2}$.

\section{Expressions for the baryon EDFFs up to NLO}

Up to NLO, which is $\mathcal{O}\left(\delta^{3}\right)$, the baryon EDFFs contain two parts: the tree-level expressions are given in table 1, and the loop contributions are obtained by taking the LO terms of the baryonic loops $J_{M M \tilde{m}}\left(q^{2}, m^{2}\right)$ and $J_{M \tilde{m}}\left(q^{2}\right)$. The explicit expressions of the EDFFs of the baryon octet up to NLO are given in the following: 
- For the neutron,

$$
\begin{aligned}
\frac{F_{3, n}\left(q^{2}\right)}{2 m_{N}}= & \frac{8}{3} e \bar{\theta}_{0}\left[\alpha w_{13}+w_{13}^{\prime r}(\mu)\right]+\frac{V_{0}^{(2)} e \bar{\theta}_{0}}{\pi^{2} F_{\pi}^{4}}\left\{( D + F ) ( b _ { D } + b _ { F } ) \left[1-\ln \frac{M_{\pi}^{2}}{\mu^{2}}+\sigma_{\pi} \ln \frac{\sigma_{\pi}-1}{\sigma_{\pi}+1}\right.\right. \\
& \left.+\frac{\pi\left(2 M_{\pi}^{2}-q^{2}\right)}{2 m_{N} \sqrt{-q^{2}}} \arctan \frac{\sqrt{-q^{2}}}{2 M_{\pi}}\right]-(D-F)\left(b_{D}-b_{F}\right)\left[1-\ln \frac{M_{K}^{2}}{\mu^{2}}+\sigma_{K} \ln \frac{\sigma_{K}-1}{\sigma_{K}+1}\right. \\
& \left.\left.+\frac{\pi}{\sqrt{-q^{2}}}\left(\frac{2 M_{K}^{2}-q^{2}}{2 m_{N}}-8\left(b_{D}-b_{F}\right)\left(M_{K}^{2}-M_{\pi}^{2}\right)\right) \arctan \frac{\sqrt{-q^{2}}}{2 M_{K}}\right]\right\},
\end{aligned}
$$

with $\alpha=144 V_{0}^{(2)} V_{3}^{(1)} /\left(F_{0} F_{\pi} M_{\eta_{0}}\right)^{2}$, and $\sigma_{\pi(K)}=\sqrt{1-4 M_{\pi(K)}^{2} / q^{2}}$. Here, we have used the $\mathrm{SU}(3)$ mass splitting for the baryon masses $m_{\Sigma}-m_{N}=4\left(b_{D}-b_{F}\right)\left(M_{K}^{2}-M_{\pi}^{2}\right)+\mathcal{O}\left(\delta^{3}\right)$. Similar relations derived from the Lagrangian eq. (2.16) will be used in the following.

- For the proton,

$$
\begin{aligned}
\frac{F_{3, p}\left(q^{2}\right)}{2 m_{N}}= & -\frac{4}{3} e \bar{\theta}_{0}\left[\alpha\left(w_{13}+3 w_{14}\right)+w_{13}^{\prime r}(\mu)+3 w_{14}^{\prime r}(\mu)\right]-\frac{V_{0}^{(2)} e \bar{\theta}_{0}}{6 \pi^{2} F_{\pi}^{4}}\left\{6(D+F)\left(b_{D}+b_{F}\right)\right. \\
& \times\left[1-\ln \frac{M_{\pi}^{2}}{\mu^{2}}+\sigma_{\pi} \ln \frac{\sigma_{\pi}-1}{\sigma_{\pi}+1}+\frac{3 \pi M_{\pi}}{2 m_{N}}+\frac{\pi\left(2 M_{\pi}^{2}-q^{2}\right)}{2 m_{N} \sqrt{-q^{2}}} \arctan \frac{\sqrt{-q^{2}}}{2 M_{\pi}}\right] \\
& +4\left(D b_{D}+3 F b_{F}\right)\left(1-\ln \frac{M_{K}^{2}}{\mu^{2}}+\sigma_{K} \ln \frac{\sigma_{K}-1}{\sigma_{K}+1}+\frac{\pi M_{K}}{m_{N}}\right) \\
& +\frac{4 \pi}{\sqrt{-q^{2}}} \arctan \frac{\sqrt{-q^{2}}}{2 M_{K}}\left[\frac{\left(D b_{D}+3 F b_{F}\right)}{2 m_{N}}\left(2 M_{K}^{2}-q^{2}\right)\right. \\
& \left.+8\left(M_{K}^{2}-M_{\pi}^{2}\right)\left(F\left(b_{D}^{2}+3 b_{F}^{2}\right)-\frac{2}{3} D b_{D}\left(b_{D}-3 b_{F}\right)\right)\right] \\
& \left.+\frac{\pi}{m_{N}}\left[6(D-F)\left(b_{D}-b_{F}\right) M_{K}+(D-3 F)\left(b_{D}-3 b_{F}\right) M_{\eta_{8}}+\frac{2 F_{\pi}^{2}}{F_{0}^{2}} \beta M_{\eta_{0}}\right]\right\},
\end{aligned}
$$

where $\beta=\left(2 D-3 w_{0}\right)\left(2 b_{D}+3 b_{0}+6 w_{10}^{\prime}\right)$.

- For the $\Sigma^{0}$,

$$
\begin{aligned}
\frac{F_{3, \Sigma^{0}}\left(q^{2}\right)}{2 m_{\Sigma}}= & -\frac{4}{3} e \bar{\theta}_{0}\left[\alpha w_{13}+w_{13}^{\prime r}(\mu)\right]-\frac{V_{0}^{(2)} e \bar{\theta}_{0}}{\pi^{2} F_{\pi}^{4}}\left\{( D b _ { F } + F b _ { D } ) \left(1-\ln \frac{M_{K}^{2}}{\mu^{2}}\right.\right. \\
& \left.+\sigma_{K} \ln \frac{\sigma_{K}-1}{\sigma_{K}+1}\right)+\frac{\pi}{\sqrt{-q^{2}}} \arctan \frac{\sqrt{-q^{2}}}{2 M_{K}}\left[\frac{D b_{F}+F b_{D}}{2 m_{\Sigma}}\left(2 M_{K}^{2}-q^{2}\right)\right. \\
& \left.\left.+8\left(M_{K}^{2}-M_{\pi}^{2}\right)\left(F b_{D}^{2}+2 D b_{D} b_{F}+F b_{F}^{2}\right)\right]\right\}
\end{aligned}
$$


- For the $\Sigma^{+}$,

$$
\begin{aligned}
\frac{F_{3, \Sigma^{+}}\left(q^{2}\right)}{2 m_{\Sigma}}= & -\frac{4}{3} e \bar{\theta}_{0}\left[\alpha\left(w_{13}+3 w_{14}\right)+w_{13}^{\prime r}(\mu)+3 w_{14}^{\prime r}(\mu)\right] \\
& -\frac{V_{0}^{(2)} e \bar{\theta}_{0}}{3 \pi^{2} F_{\pi}^{4}}\left\{2\left(D b_{D}+3 F b_{F}\right)\left(1-\ln \frac{M_{\pi}^{2}}{\mu^{2}}+\sigma_{\pi} \ln \frac{\sigma_{\pi}-1}{\sigma_{\pi}+1}+\frac{\pi M_{\pi}}{m_{\Sigma}}\right)\right. \\
& +3(D+F)\left(b_{D}+b_{F}\right)\left[1-\ln \frac{M_{K}^{2}}{\mu^{2}}+\sigma_{K} \ln \frac{\sigma_{K}-1}{\sigma_{K}+1}+\frac{\pi M_{K}}{m_{\Sigma}}\right. \\
& \left.+\frac{\pi}{\sqrt{-q^{2}}}\left(\frac{2 M_{K}^{2}-q^{2}}{2 m_{\Sigma}}+8\left(b_{D}+b_{F}\right)\left(M_{K}^{2}-M_{\pi}^{2}\right)\right) \arctan \frac{\sqrt{-q^{2}}}{2 M_{K}}\right] \\
& +\frac{2 \pi}{\sqrt{-q^{2}}} \arctan \frac{\sqrt{-q^{2}}}{2 M_{\pi}}\left[\frac{\left(D b_{D}+3 F b_{F}\right)}{2 m_{\Sigma}}\left(2 M_{\pi}^{2}-q^{2}\right)+\frac{32}{3} D b_{D}^{2}\left(M_{K}^{2}-M_{\pi}^{2}\right)\right] \\
& \left.+\frac{\pi}{m_{\Sigma}}\left[6 F b_{F} M_{\pi}+3(D-F)\left(b_{D}-b_{F}\right) M_{K}+2 D b_{D} M_{\eta_{8}}+\frac{F_{\pi}^{2}}{F_{0}^{2}} \beta M_{\eta_{0}}\right]\right\} .(\mathrm{C} .4)
\end{aligned}
$$

- For the $\Sigma^{-}$,

$$
\begin{aligned}
\frac{F_{3, \Sigma^{-}}\left(q^{2}\right)}{2 m_{\Sigma}}= & -\frac{4}{3} e \bar{\theta}_{0}\left[\alpha\left(w_{13}-3 w_{14}\right)+w_{13}^{\prime r}(\mu)-3 w_{14}^{\prime r}(\mu)\right] \\
& +\frac{V_{0}^{(2)} e \bar{\theta}_{0}}{3 \pi^{2} F_{\pi}^{4}}\left\{2\left(D b_{D}+3 F b_{F}\right)\left(1-\ln \frac{M_{\pi}^{2}}{\mu^{2}}+\sigma_{\pi} \ln \frac{\sigma_{\pi}-1}{\sigma_{\pi}+1}+\frac{\pi M_{\pi}}{m_{\Sigma}}\right)\right. \\
& +\frac{2 \pi}{\sqrt{-q^{2}}} \arctan \frac{\sqrt{-q^{2}}}{2 M_{\pi}}\left[\frac{\left(D b_{D}+3 F b_{F}\right)}{2 m_{\Sigma}}\left(2 M_{\pi}^{2}-q^{2}\right)+\frac{32}{3} D b_{D}^{2}\left(M_{K}^{2}-M_{\pi}^{2}\right)\right] \\
& +3(D-F)\left(b_{D}-b_{F}\right)\left[1-\ln \frac{M_{K}^{2}}{\mu^{2}}+\sigma_{K} \ln \frac{\sigma_{K}-1}{\sigma_{K}+1}+\frac{\pi M_{K}}{m_{\Sigma}}\right. \\
& \left.+\frac{\pi}{\sqrt{-q^{2}}}\left(\frac{2 M_{K}^{2}-q^{2}}{2 m_{\Sigma}}+8\left(b_{D}-b_{F}\right)\left(M_{K}^{2}-M_{\pi}^{2}\right)\right) \arctan \frac{\sqrt{-q^{2}}}{2 M_{K}}\right] \\
& \left.+\frac{\pi}{m_{\Sigma}}\left[6 F b_{F} M_{\pi}+3(D+F)\left(b_{D}+b_{F}\right) M_{K}+2 D b_{D} M_{\eta_{8}}+\frac{F_{\pi}^{2}}{F_{0}^{2}} \beta M_{\eta_{0}}\right]\right\} . \text { (C.5) }
\end{aligned}
$$

- For the $\Lambda$

$$
\begin{aligned}
\frac{F_{3, \Lambda}\left(q^{2}\right)}{2 m_{\Lambda}}= & \frac{4}{3} e \bar{\theta}_{0}\left[\alpha w_{13}+w_{13}^{\prime r}(\mu)\right]+\frac{V_{0}^{(2)} e \bar{\theta}_{0}}{\pi^{2} F_{\pi}^{4}}\left\{\left(D b_{F}+F b_{D}\right)\left(1-\ln \frac{M_{K}^{2}}{\mu^{2}}+\sigma_{K} \ln \frac{\sigma_{K}-1}{\sigma_{K}+1}\right)\right. \\
& +\frac{\pi}{\sqrt{-q^{2}}} \arctan \frac{\sqrt{-q^{2}}}{2 M_{K}}\left[\frac{D b_{F}+F b_{D}}{2 m_{\Sigma}}\left(2 M_{K}^{2}-q^{2}\right)\right. \\
& \left.\left.-\frac{8}{3}\left(M_{K}^{2}-M_{\pi}^{2}\right)\left(F b_{D}^{2}+2 D b_{D} b_{F}+F b_{F}^{2}\right)\right]\right\} .
\end{aligned}
$$


- For the $\Xi^{0}$,

$$
\begin{aligned}
\frac{F_{3, \Xi^{0}}\left(q^{2}\right)}{2 m_{\Xi}}= & \frac{8}{3} e \bar{\theta}_{0}\left[\alpha w_{13}+w_{13}^{\prime r}(\mu)\right]-\frac{V_{0}^{(2)} e \bar{\theta}_{0}}{\pi^{2} F_{\pi}^{4}}\left\{( D - F ) ( b _ { D } - b _ { F } ) \left[1-\ln \frac{M_{\pi}^{2}}{\mu^{2}}+\sigma_{\pi} \ln \frac{\sigma_{\pi}-1}{\sigma_{\pi}+1}\right.\right. \\
& \left.+\frac{\pi\left(2 M_{\pi}^{2}-q^{2}\right)}{2 m_{\Xi} \sqrt{-q^{2}}} \arctan \frac{\sqrt{-q^{2}}}{2 M_{\pi}}\right]-(D+F)\left(b_{D}+b_{F}\right)\left[1-\ln \frac{M_{K}^{2}}{\mu^{2}}+\sigma_{K} \ln \frac{\sigma_{K}-1}{\sigma_{K}+1}\right. \\
& \left.\left.+\frac{\pi}{\sqrt{-q^{2}}}\left(\frac{2 M_{K}^{2}-q^{2}}{2 m_{\Xi}}-8\left(b_{D}+b_{F}\right)\left(M_{K}^{2}-M_{\pi}^{2}\right)\right) \arctan \frac{\sqrt{-q^{2}}}{2 M_{K}}\right]\right\},
\end{aligned}
$$

- For the $\Xi^{-}$,

$$
\begin{aligned}
\frac{F_{3, \Xi^{-}}\left(q^{2}\right)}{2 m_{\Xi}}= & -\frac{4}{3} e \bar{\theta}_{0}\left[\alpha\left(w_{13}-3 w_{14}\right)+w_{13}^{\prime r}(\mu)-3 w_{14}^{\prime r}(\mu)\right]+\frac{V_{0}^{(2)} e \bar{\theta}_{0}}{6 \pi^{2} F_{\pi}^{4}}\left\{6(D-F)\left(b_{D}-b_{F}\right)\right. \\
& \times\left[1-\ln \frac{M_{\pi}^{2}}{\mu^{2}}+\sigma_{\pi} \ln \frac{\sigma_{\pi}-1}{\sigma_{\pi}+1}+\frac{3 \pi M_{\pi}}{2 m_{\Xi}}+\frac{\pi\left(2 M_{\pi}^{2}-q^{2}\right)}{2 m_{\Xi} \sqrt{-q^{2}}} \arctan \frac{\sqrt{-q^{2}}}{2 M_{\pi}}\right] \\
& +4\left(D b_{D}+3 F b_{F}\right)\left(1-\ln \frac{M_{K}^{2}}{\mu^{2}}+\sigma_{K} \ln \frac{\sigma_{K}-1}{\sigma_{K}+1}+\frac{\pi M_{K}}{m_{\Xi}}\right) \\
& +\frac{4 \pi}{\sqrt{-q^{2}}} \arctan \frac{\sqrt{-q^{2}}}{2 M_{K}}\left[\frac{D b_{D}+3 F b_{F}}{2 m_{\Xi}}\left(2 M_{K}^{2}-q^{2}\right)\right. \\
& \left.-8\left(M_{K}^{2}-M_{\pi}^{2}\right)\left(F\left(b_{D}^{2}+3 b_{F}^{2}\right)+\frac{2}{3} D b_{D}\left(b_{D}+3 b_{F}\right)\right)\right] \\
& \left.+\frac{\pi}{m_{\Xi}}\left[6(D+F)\left(b_{D}+b_{F}\right) M_{K}+(D+3 F)\left(b_{D}+3 b_{F}\right) M_{\eta_{8}}+\frac{2 F_{\pi}^{2}}{F_{0}^{2}} \beta M_{\eta_{0}}\right]\right\} .
\end{aligned}
$$

The expressions for the EDMs can be easily obtained by noticing

$$
\lim _{q^{2} \rightarrow 0} \sigma \ln \frac{\sigma-1}{\sigma+1}=-2, \quad \lim _{q^{2} \rightarrow 0} \frac{1}{\sqrt{-q^{2}}} \arctan \frac{\sqrt{-q^{2}}}{2 M}=\frac{1}{2 M} .
$$

\section{Finite volume corrections to loops}

This appendix is dedicated to finite volume corrections to the two- and three-point loop integrals. Let us consider the scalar two-point loop

$$
\begin{aligned}
J_{m_{1} m_{2}}\left(q^{2}\right) & =i \int \frac{d^{4} k}{(2 \pi)^{4}} \frac{1}{\left(k^{2}-m_{1}^{2}+i \epsilon\right)\left[(k+q)^{2}-m_{2}^{2}+i \epsilon\right]} \\
& =\int_{0}^{1} d x i \int \frac{d^{4} k}{(2 \pi)^{4}} \frac{1}{\left(k^{2}-\bar{m}_{12}^{2}+i \epsilon\right)^{2}}
\end{aligned}
$$

where $\bar{m}_{12}^{2}=x(x-1) q^{2}+(1-x) m_{1}^{2}+x m_{2}^{2}$. If the loop involves both a meson and a baryon, $m_{1}$ should be replaced by the mesonic mass, and the upper bound of the integration over the Feynman 
parameter $x$ should be replaced by $\infty$ in infrared regularization. Performing the contour integral over $k^{0}$, one gets

$$
J_{m_{1} m_{2}}\left(q^{2}\right)=-\frac{1}{4} \int_{0}^{1} d x \int \frac{d^{3} \vec{k}}{(2 \pi)^{3}} \frac{1}{\left(\vec{k}^{2}+\bar{m}_{12}^{2}\right)^{3 / 2}} .
$$

Using the formula

$$
\left(\frac{1}{L^{3}} \sum_{\vec{n}}-\int \frac{d^{3} \vec{k}}{(2 \pi)^{3}}\right) \frac{1}{\left(\vec{k}^{2}+a^{2}\right)^{j}}=\frac{2^{-j} a^{3-2 j}}{\sqrt{2} \pi^{3 / 2} \Gamma(j)} \sum_{\vec{n} \neq 0} \frac{K_{3 / 2-j}(L a|\vec{n}|)}{(L a|\vec{n}|)^{3 / 2-j}}
$$

derived in ref. [52], we get the finite volume correction to the loop $J_{m_{1} m_{2}}\left(q^{2}\right)$,

$$
\delta_{L}\left[J_{m_{1} m_{2}}\left(q^{2}\right)\right]=-\frac{1}{8 \pi^{2}} \int_{0}^{1} d x \sum_{\vec{n} \neq 0} K_{0}\left(L \bar{m}_{12}|\vec{n}|\right),
$$

with $K_{\nu}(z)$ a modified Bessel function of the second kind.

From eq. (B.4), it is easy to write down the correction to the three-point loop integral,

$$
\begin{aligned}
\delta_{L}\left[J_{M M m}\left(q^{2}, p^{2}\right)\right] & =\frac{\partial}{\partial M^{2}} \int_{0}^{1} d x \delta_{L}\left[J_{\bar{M} m}\left(\bar{p}^{2}\right)\right] \\
& =\frac{L^{2}}{16 \pi^{2}} \sum_{\vec{n} \neq 0} \vec{n}^{2} \int_{0}^{1} d x \int_{0}^{\infty} d y \frac{1-y}{z_{1}} K_{1}\left(z_{1}\right),
\end{aligned}
$$

where $z_{1}=L|\vec{n}|\left[y(y-1) \bar{p}^{2}+y m^{2}+(1-y) \bar{M}^{2}\right]^{1 / 2}$. We have made use of the integral representation of $K_{n}(z)$ [53]

$$
K_{n}(z)=\frac{\Gamma(n+1 / 2)(2 z)^{n}}{\sqrt{\pi}} \int_{0}^{\infty} d t \frac{\cos t}{\left(t^{2}+z^{2}\right)^{n+1 / 2}} .
$$

which is valid for $\operatorname{Re} n>-1 / 2,|\arg z|<\pi / 2$. Similarly, one gets

$$
\delta_{L}\left[J_{M m m}\left(p^{2}, p^{2}\right)\right]=\frac{L^{2}}{16 \pi^{2}} \sum_{\vec{n} \neq 0} \vec{n}^{2} \int_{0}^{1} d x \int_{0}^{\infty} d y \frac{y}{z_{2}} K_{1}\left(z_{2}\right),
$$

where $z_{2}=L|\vec{n}|\left[y(y-1) \bar{p}^{2}+y \bar{m}^{2}+(1-y) M^{2}\right]^{1 / 2}$.

\section{E Asymptotic expansion of finite volume corrections}

For simplicity, we will focus on the asymptotic expansion of finite volume corrections with $q^{2}=0$ and $p^{2}=m^{2}$. The general case can be treated similarly. For $|z| \rightarrow \infty$, one has

$$
K_{n}(z) \sim \sqrt{\frac{\pi}{2 z}} e^{-z}\left[1+\mathcal{O}\left(\frac{1}{z}\right)\right] .
$$

For $L \rightarrow \infty$, we can consider the term with $|\vec{n}|=1$ only in eq. (D.4). There are 6 possibilities, so that for $L \rightarrow \infty$,

$$
\delta_{L}\left[J_{M M}(0)\right] \sim-\frac{3}{4 \pi^{2}} \sqrt{\frac{\pi}{2}} \frac{e^{-L M}}{\sqrt{L M}}
$$


For the three-point loop integral,

$$
\delta_{L}\left[J_{M M m}\left(0, m^{2}\right)\right] \sim \frac{L^{2}}{16 \pi^{2}} \sqrt{\frac{\pi}{2}} \int_{0}^{\infty} d y(1-y) \frac{e^{-L C(y)}}{[L C(y)]^{3 / 2}},
$$

with $C(y)=\left[y^{2} m^{2}+(1-y) M^{2}\right]^{1 / 2}$. The leading term of the above integral over the Feynman parameter $y$ can be worked out using Laplace's method. For very large $L$, the integral receives contributions mostly from the neighborhood of the minimum of $C(y)$, which is at $y_{c}=M^{2} /\left(2 m^{2}\right)$. Thus,

$$
\begin{aligned}
\delta_{L}\left[J_{M M m}\left(0, m^{2}\right)\right] & \sim \frac{3 L^{2}}{8 \pi^{2}} \sqrt{\frac{\pi}{2}}\left(1-y_{c}\right) \frac{e^{-L C\left(y_{c}\right)}}{\left[L C\left(y_{c}\right)\right]^{3 / 2}} \int_{-\infty}^{\infty} d y \exp \left[-\frac{1}{2} y^{2} C^{\prime \prime}\left(y_{c}\right)\right] \\
& =\frac{3}{8 \pi m M} \exp \left(-L M \sqrt{1-\frac{M^{2}}{4 m^{2}}}\right)\left[1+\mathcal{O}\left(\frac{M^{2}}{m^{2}}\right)\right] .
\end{aligned}
$$

The asymptotic expansion for $\delta_{L}\left[J_{M m m}\left(m^{2}, m^{2}\right)\right]$ is the same.

\section{References}

[1] C. A. Baker et al., An improved experimental limit on the electric dipole moment of the neutron, Phys. Rev. Lett. 97 (2006) 131801 [hep-ex/0602020].

[2] M. Pospelov and A. Ritz, Electric dipole moments as probes of new physics, Annals Phys. 318 (2005) 119 [hep-ph/0504231].

[3] S. K. Lamoreaux and R. Golub, Experimental searches for the neutron electric dipole moment, J. Phys. G 36 (2009) 104002.

[4] F. J. M. Farley, K. Jungmann, J. P. Miller, W. M. Morse, Y. F. Orlov, B. L. Roberts, Y. K. Semertzidis and A. Silenko et al., A New method of measuring electric dipole moments in storage rings, Phys. Rev. Lett. 93 (2004) 052001 [hep-ex/0307006].

[5] Y. K. Semertzidis [Storage Ring EDM Collaboration], A Storage Ring proton Electric Dipole Moment experiment: most sensitive experiment to CP-violation beyond the Standard Model, arXiv:1110.3378 [physics.acc-ph].

[6] F. Rathmann and N. Nikolaev, Precursor experiments to search for permanent electric dipole moments (EDMs) of protons and deuterons at COSY, PoS STORI11 (2011) 029.

[7] A. Lehrach, B. Lorentz, W. Morse, N. Nikolaev and F. Rathmann, Precursor experiments to search for permanent electric dipole moments (EDMs) of protons and deuterons at COSY, arXiv:1201.5773 [hep-ex].

[8] S. Aoki and A. Gocksch, The neutron electric dipole moment in lattice QCD, Phys. Rev. Lett. 63 (1989) 1125 [Erratum ibid 65 (1990) 1172].

[9] S. Aoki, A. Gocksch, A. V. Manohar and S. R. Sharpe, Calculating the neutron electric dipole moment on the lattice, Phys. Rev. Lett. 65 (1990) 1092.

[10] E. Shintani, S. Aoki, N. Ishizuka, K. Kanaya, Y. Kikukawa, Y. Kuramashi, M. Okawa and A. Ukawa et al., Neutron electric dipole moment with external electric field method in lattice QCD, Phys. Rev. D 75 (2007) 034507 [hep-lat/0611032]. 
[11] E. Shintani, S. Aoki and Y. Kuramashi, Full QCD calculation of neutron electric dipole moment with the external electric field method, Phys. Rev. D 78 (2008) 014503 [arXiv:0803.0797 [hep-lat]].

[12] E. Shintani, S. Aoki, N. Ishizuka, K. Kanaya, Y. Kikukawa, Y. Kuramashi, M. Okawa and Y. Tanigchi et al., Neutron electric dipole moment from lattice QCD, Phys. Rev. D 72 (2005) 014504 [hep-lat/0505022].

[13] F. Berruto, T. Blum, K. Orginos and A. Soni, Calculation of the neutron electric dipole moment with two dynamical flavors of domain wall fermions, Phys. Rev. D 73 (2006) 054509 [hep-lat/0512004].

[14] T. Izubuchi, S. Aoki, K. Hashimoto, Y. Nakamura, T. Sekido and G. Schierholz, Dynamical QCD simulation with theta terms, PoS LAT 2007 (2007) 106 [arXiv:0802.1470 [hep-lat]].

[15] S. Aoki, R. Horsley, T. Izubuchi, Y. Nakamura, D. Pleiter, P. E. L. Rakow, G. Schierholz and $\mathrm{J}$. Zanotti, The Electric dipole moment of the nucleon from simulations at imaginary vacuum angle theta, arXiv:0808.1428 [hep-lat].

[16] E. Shintani, talk given at the Xth Quark Confinement and the Hadron Spectrum, Garching, Oct. 8-12, 2012.

[17] G. Schierholz, talk given at the ECT* Workshop on EDM Searches at Storage Rings, Trento, Oct. $2-5,2012$

[18] S. J. Brodsky, S. Gardner and D. S. Hwang, Discrete symmetries on the light front and a general relation connecting nucleon electric dipole and anomalous magnetic moments, Phys. Rev. D 73 (2006) 036007 [hep-ph/0601037].

[19] K.-F. Liu, Neutron electric dipole moment at fixed topology, Mod. Phys. Lett. A 24 (2009) 1971 [arXiv:0807.1365 [hep-ph]].

[20] E. Mereghetti, W. H. Hockings and U. van Kolck, The effective chiral Lagrangian from the theta term, Annals Phys. 325 (2010) 2363 [arXiv:1002.2391 [hep-ph]].

[21] S. D. Thomas, Electromagnetic contributions to the Schiff moment, Phys. Rev. D 51 (1995) 3955 [arXiv:hep-ph/9402237].

[22] B. Borasoy, The electric dipole moment of the neutron in chiral perturbation theory, Phys. Rev. D 61 (2000) 114017 [arXiv:hep-ph/0004011].

[23] R. J. Crewther, P. Di Vecchia, G. Veneziano and E. Witten, Chiral estimate of the electric dipole moment of the neutron in quantum chromodynamics, Phys. Lett. B 88 (1979) 123 [Erratum ibid B 91 (1980) 487].

[24] A. Pich and E. de Rafael, Strong CP violation in an effective chiral Lagrangian approach, Nucl. Phys. B 367 (1991) 313.

[25] S. Narison, A fresh look into the neutron EDM and magnetic susceptibility, Phys. Lett. B 666 (2008) 455 [arXiv:0806.2618 [hep-ph]].

[26] W. H. Hockings and U. van Kolck, The electric dipole form factor of the nucleon, Phys. Lett. B 605 (2005) 273 [arXiv:nucl-th/0508012].

[27] K. Ottnad, B. Kubis, U.-G. Meißner and F.-K. Guo, New insights into the neutron electric dipole moment, Phys. Lett. B 687 (2010) 42 [arXiv:0911.3981 [hep-ph]].

[28] E. Mereghetti, J. de Vries, W. H. Hockings, C. M. Maekawa and U. van Kolck, The electric dipole form factor of the nucleon in chiral perturbation theory to sub-leading order, Phys. Lett. B 696 (2011) 97 [arXiv:1010.4078 [hep-ph]]. 
[29] D. O'Connell and M. J. Savage, Extrapolation formulas for neutron EDM calculations in lattice QCD, Phys. Lett. B 633 (2006) 319 [hep-lat/0508009].

[30] J.-W. Chen, D. O'Connell and A. Walker-Loud, Universality of mixed action extrapolation formulae, JHEP 04 (2009) 090 [arXiv:0706.0035 [hep-lat]].

[31] J. F. Donoghue, E. Golowich and B. R. Holstein, Dynamics of the Standard Model, Cambridge University Press, Cambridge (1992).

[32] J. Gasser and H. Leutwyler, Chiral perturbation theory: Expansions in the mass of the strange quark, Nucl. Phys. B 250 (1985) 465.

[33] H. Leutwyler, Bounds on the light quark masses, Phys. Lett. B 374 (1996) 163 [arXiv:hep-ph/9601234].

[34] P. Herrera-Siklody, J. I. Latorre, P. Pascual and J. Taron, Chiral effective Lagrangian in the large- $N_{c}$ limit: The nonet case, Nucl. Phys. B 497 (1997) 345 [arXiv:hep-ph/9610549].

[35] K. Ottnad, The electric dipole form factor of the neutron in chiral perturbation theory, Diploma thesis, University of Bonn (2009).

[36] T. Becher and H. Leutwyler, Baryon chiral perturbation theory in manifestly Lorentz invariant form, Eur. Phys. J. C 9 (1999) 643 [hep-ph/9901384].

[37] B. Borasoy and U.-G. Meißner, Chiral expansion of baryon masses and sigma terms, Annals Phys. 254 (1997) 192 [hep-ph/9607432].

[38] J. Bsaisou, C. Hanhart, S. Liebig, U.-G. Meißner, A. Nogga and A. Wirzba, The electric dipole moment of the deuteron from the QCD $\theta$-term, arXiv:1209.6306 [hep-ph].

[39] J. Beringer et al. [Particle Data Group], Review of Particle Physics, Phys. Rev. D 86 (2012) 010001.

[40] P. Herrera-Siklody, J. I. Latorre, P. Pascual and J. Taron, $\eta-\eta^{\prime}$ mixing from $U(3)_{L} \otimes U(3)_{R}$ chiral perturbation theory, Phys. Lett. B 419 (1998) 326 [hep-ph/9710268].

[41] B. C. Tiburzi, External momentum, volume effects, and the nucleon magnetic moment, Phys. Rev. D 77 (2008) 014510 [arXiv:0710.3577 [hep-lat]].

[42] L. Greil, T. R. Hemmert and A. Schafer, Finite Volume Corrections to the Electromagnetic Current of the Nucleon, Eur. Phys. J. A 48 (2012) 53 [arXiv:1112.2539 [hep-ph]].

[43] J. Gasser and H. Leutwyler, Spontaneously broken symmetries: Effective lagrangians at finite volume, Nucl. Phys. B 307 (1988) 763.

[44] L.-s. Geng, X.-1. Ren, J. Martin-Camalich and W. Weise, Finite-volume effects on octet-baryon masses in covariant baryon chiral perturbation theory, Phys. Rev. D 84 (2011) 074024 [arXiv:1108.2231 [hep-ph]].

[45] J. Gasser and H. Leutwyler, Light quarks at low temperatures, Phys. Lett. B 184 (1987) 83.

[46] B. C. Tiburzi, Volume effects for pion two-point functions in constant electric and magnetic fields, Phys. Lett. B 674 (2009) 336 [arXiv:0809.1886 [hep-lat]].

[47] J. Gasser, M. E. Sainio and A. Švarc, Nucleons with chiral loops, Nucl. Phys. B 307 (1988) 779.

[48] E. E. Jenkins and A. V. Manohar, Baryon chiral perturbation theory using a heavy fermion Lagrangian, Phys. Lett. B 255 (1991) 558.

[49] V. Bernard, N. Kaiser and U.-G. Meißner, Chiral dynamics in nucleons and nuclei, Int. J. Mod. Phys. E 4 (1995) 193 [hep-ph/9501384]. 
[50] V. Bernard, Chiral perturbation theory and baryon properties, Prog. Part. Nucl. Phys. 60 (2008) 82 [arXiv:0706.0312 [hep-ph]].

[51] P. J. Ellis and H.-B. Tang, Pion nucleon scattering in a new approach to chiral perturbation theory, Phys. Rev. C 57 (1998) 3356 [hep-ph/9709354].

[52] S. R. Beane, Nucleon masses and magnetic moments in a finite volume, Phys. Rev. D 70 (2004) 034507 [hep-lat/0403015].

[53] M. Abramowitz and I. A. Stegun, Handbook of Mathematical Functions with Formulas, Graphs, and Mathematical Tables, Dover, New York (1972). 